\title{
The stationary banding complex and secondary eyewall formation in tropical cyclones
}

\author{
Anna Vaughan ${ }^{1 *}$, Kevin J. E. Walsh ${ }^{1}$, Jeffrey D. Kepert ${ }^{2}$ \\ ${ }^{1}$ School of Earth Sciences, University of Melbourne, Victoria, Australia \\ ${ }^{2}$ Bureau of Meteorology, Melbourne, Victoria, Australia
}

\section{Key Points:}

- A stationary banding complex is observed in $39 \%$ of passive microwave images of tropical cyclones.

- A stationary banding complex is observed within 6 hours of secondary eyewall formation in $79 \%$ of events.

- Stationary banding complex crossing angle (azimuthal extent) is significantly lower (higher) within 12 hours of secondary eyewall formation.

This is the author manuscript accepted for publication and has undergone full peer review but has not been through the copyediting, typesetting, pagination and proofreading process, which may lead to differences between this version and the Version of Record. Please cite this article as dor: $10.1029,2019$ J 2031515 of Earth Sciences, University of Cambridge, Cambridge, UK

Corresponding author: Anna Vaughan, av555@cam.ac.uk

This article is protected by copyright. All rights reserved. 


\begin{abstract}
$89 \mathrm{GHz}$ passive microwave satellite data is used to develop a five year climatology of the incidence and morphology of the stationary banding complex in tropical cyclones and quantify changes in convective morphology prior to secondary eyewall formation. The stationary banding complex is shown to be present in $39 \%$ of passive microwave overpasses. Morphology varies substantially between tropical cyclones, with crossing angles ranging from 0.19 to 61.78 degrees and azimuthal extents from $0.29 \pi$ to $4.02 \pi$ radians. Variations in the incidence and geometry of the stationary banding complex are observed in different environmental conditions and geographic locations. For 84 secondary eyewall formation events included in the sample, a stationary banding complex is observed within 6 hours of the secondary eyewall developing in $79 \%$ of cases. Within 12 hours prior to secondary eyewall formation, the crossing angle is significantly lower than its sample median, while the azimuthal extent is higher than its sample median. These results demonstrate that secondary eyewall formation is (most) often preceded by the formation and axisymmetrisation of a stationary banding complex.
\end{abstract}

\title{
1 Introduction
}

Understanding the incidence and dynamics of variations in the convective structure of tropical cyclones (TCs) is vital for improving forecasting of intensity $(?, ?, ?)$, size $(?$, ?), storm surge (?, ?), wind radii (?, ?) and surface wave height (?, ?). Two frequently observed changes in convective morphology are Secondary Eyewall Formation (SEF; ?, ?) and the development of a stationary banding complex (SBC; ?, ?). SEF occurs when outer rainbands coalesce to form a new eyewall concentric to the existing inner eyewall, known as a secondary eyewall (SE). An SBC is a mesoscale wavenumber one convective asymmetry that remains quasi-stationary relative to the vortex. This asymmetry consists of one dominant rainband known as the principal rainband, which is separated from the inner core by a convection free moat. Smaller rainbands termed the secondary rainbands may be joined to the edges of the principal rainband. Schematics of these configurations are shown in Figure 1.

During SEF, tropical cyclones often exhibit increases in size (?, ?, ?) and integrated kinetic energy $(?, ?)$, and often changes in intensity also $(?, ?, ?)$. Understanding this process is therefore vital from the perspective of operational forecasting, yet SEF remains difficult to predict $(?, ?, ?, ?)$, and the dynamical processes responsible for the development of the SE are not well understood (?, ?).

It is well established that the SE forms from convection in pre-existing spiral rainbands (e.g ?, ?). Numerical simulations suggest that SEF is initiated by projection of rainband diabatic heating onto the azimuthal mean (?, ?), vorticity accumulation in the outer rainbands $(?, ?)$ or boundary layer feedbacks in response to rainband heating and vorticity anomalies (?, ?, ?, ?). Case studies of SEF events in hurricanes Rita (2005) (?, ?, ?, ?, ?) and Earl (2010) (?, ?) used high resolution Doppler radar observations to demonstrate that, in both of these cases, an SE developed from the axisymmetrisation of a pre-

This article is protected by copyright. All rights reserved. 


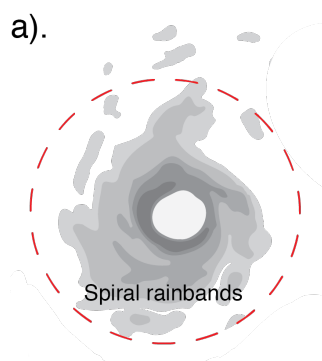

b).

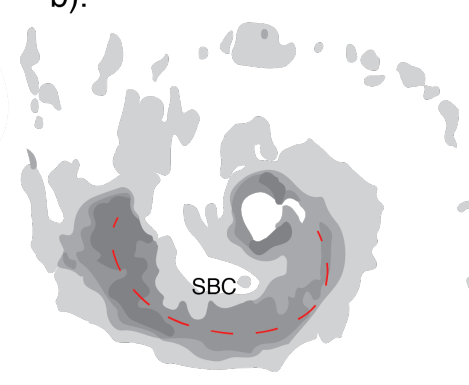

c).

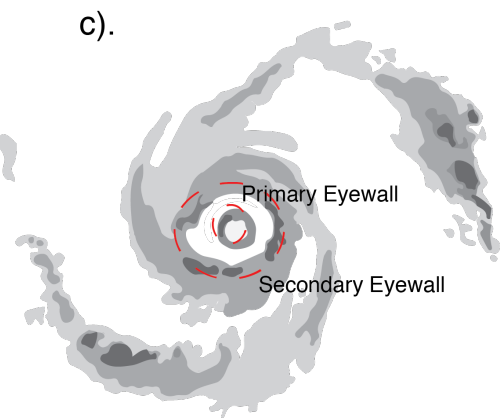

Figure 1. Schematic diagrams of $89 \mathrm{GHz}$ brightness temperature illustrating different convective morphology in TCs, showing (a) spiral banding, (b) a stationary banding complex (SBC) and (c) concentric eyewalls.

existing SBC. For both of these events, a strong mid-level mesoscale inflow jet developed in the downshear-left quadrant, descending into the boundary layer and creating a local vorticity maximum at the radius of the incipient SE. ? (?) suggested that this jet acts to accelerate the tangential circulation in the downshear left quadrant, after which SEF is triggered by axisymmetric processes. ? (?) observed a similar inflow pattern in a numerical simulation, suggesting that this operates in conjunction with axisymmetric boundary layer mechanisms to trigger SEF by enhancing inflow on the opposite side of the TC. This pathway to SEF has been observed in other numerical simulations (?, ?, ?).

Together, this previous work strongly suggests a relationship between the SBC and SEF. Despite this, it remains unclear how rainband morphology varies prior to SEF climatologically. Quantifying this variation is difficult as no previous work has examined the climatological incidence or morphology of the SBC. It is well understood that the $\mathrm{SBC}$ is oriented relative to the direction of the deep layer environmental shear, with convective cells developing in the downshear right quadrant, propagating down the band and dissipating in the downshear left quadrant (e.g ?, ?, ?). SBC formation has been attributed to shear induced asymmetries in potential temperature and vorticity induced by the vortex tilt $(?, ?, ?, ?)$. Understanding of the structure and morphology of individual bands is derived either from case studies (?, ?, ?, ?, ?, ?, ?), or composite profiles of microphysical structure that do not capture the morphology of individual bands (?, ?, ?). To date, studies of rainband geometry have been limited to case studies of individual rainbands using airborne Doppler radar (?, ?, ?, ?, ?), or small samples of rainbands using land based radar (?, ?).

This study aims to clarify the role of the SBC in SEF by developing the first climatology of SBC formation and morphology. Specifically, we aim to:

1. Quantify the climatological incidence of SBC formation, and document how this varies geographically. 
2. Quantify variations in SBC morphology.

3. Establish the incidence of the SBC and variations in morphology at different times prior to SEF.

Section 2 describes the dataset used to identify SBCs and SEs and the analysis methodology. Section 3 presents a climatology of SBC incidence and formation, with morphology discussed in section 4 . These results are used in section 5 to quantify changes in rainband morphology prior to SEF. Finally, a discussion and conclusions are presented in Section 6. Methodology and results are based on those outlined by ? (?).

\section{Dataset and methodology}

\subsection{Dataset}

\subsubsection{Passive microwave dataset}

A five year TC centred Passive Microwave (PMW) dataset is used to identify SEs and SBCs. Raw $85-92 \mathrm{GHz}$ data is taken from the NASA Precipitation Processing System STORM archive (?, ?) using level 1C products for the Global Precipitation Mission Microwave Imager (GMI; ?, ?), Advanced Microwave Scanning Radiometer 2 (AMSR2; ?, ?), and the Special Sensor Microwave Imager/Sounder (SSMIS; ?, ?). 85-92GHz data is suitable for viewing convective morphology as this frequency range is unaffected by the upper level cirrus canopy that obscures infared observations (?, ?), and has been used to identify SEs in previous climatologies (?, ?, ?, ?).

A limitation of PMW imagery is that no PMW instruments are in geostationary orbit, resulting in irregular spatial and temporal coverage. Together, the constellation of instruments used here gives a mean revisit time of 4.20 hours for this dataset. Given that the structure of an SBC evolves on a timescale of at least 6 hours $(?, ?, ?)$ and SEs on a timescale of at least 12 hours $(?, ?)$, this temporal resolution is sufficient for this study. Swath widths for these six instruments range from $930-1700 \mathrm{~km}$, sufficient to cover the entire rainband field in a majority of cases.

For each swath in the raw dataset, the International Best Tracks for Climate Stewardship (IBTrACS; ?, ?) dataset is used to identify any TCs with a centre fix within the swath and intensity greater than $65 \mathrm{kts}$ at the time of the satellite overpass. Both the horizontally and vertically polarised brightness temperature data for each TC contained within the swath are regridded to a $1500 \times 1500 \mathrm{~km}$ Cartesian grid centred on the TC at $2 \mathrm{~km}$ resolution using bi-cubic spline interpolation. The choice of $2 \mathrm{~km}$ resolution is made to be consistent with other PMW image archives, which have a resolution of $1-2 \mathrm{~km}$ (?, ?, ?). It is noted that all instruments are recalibrated to $89 \mathrm{GHz}$ using histogram matching in the raw dataset (?, ?), eliminating uncertainty due to frequency differences $(?, ?)$.

False colour composites are produced from the raw channels following a technique similar to that outlined by (?, ?). The red (R), blue (B) and green (G) channels of the $89 \mathrm{GHz}$ satellite images are determined according to the following equations. 


$$
R=\left(290-P C T_{89}\right) \times \frac{255}{130}, \quad R\left(P C T_{89}<160\right)=255 \quad R\left(P C T_{89}>290\right)=0
$$

$$
G=255-\left(290-89_{v p o l}\right) \times \frac{255}{20}, \quad G\left(89_{v p o l}<270\right)=0 \quad G\left(89_{v p o l}>290\right)=255
$$

$$
B=255-\left(290-89_{\text {hpol }}\right) \times \frac{255}{130}, \quad B\left(89_{\text {hpol }}<160\right)=0 \quad B\left(89_{\text {hpol }}>290\right)=255
$$

where $89_{v p o l}$ is the $89 \mathrm{GHz}$ vertically polarised brightness temperature, $89_{\text {hpol }}$ is the $89 \mathrm{GHz}$ horizontally polarised brightness temperature channel and $P C T_{89}$ is the $89 \mathrm{GHz}$ polarisation corrected temperature (PCT), defined by (Spencer et al. 1989):

$$
P C T_{89}=1.818 \times 89_{\text {vpol }}+0.818 \times 89_{\text {hpol }}
$$

Identifying the extent of rainbands relies on determining which pixels in each image are classified as raining. As a $P C T_{89}$ of less than $255^{\circ} \mathrm{K}$ has been established a threshold for precipitation (Spencer et al., 1989), a further constraint is imposed that

$$
R\left(P C T_{89}<255^{\circ} \mathrm{K}\right)=0
$$

Examples of the resulting PMW images are shown in Figure 2. In these images, red pixels indicate areas of rain, while green and blue indicate low level warm clouds or the underlying surface. Prior to classification, each image is manually checked and rejected if the entire rainband field is not included in the swath or any part of the TC is over land.

\subsubsection{Environmental conditions}

To quantify the large scale environmental conditions at the time of each image, the current maximum intensity, maximum potential intensity and deep layer (850-200hPa) shear magnitude are linearly interpolated to the time of each overpass. Maximum intensity data is taken from the IBTrACS dataset (?, ?). Maximum potential intensity and deep layer shear magnitude are taken from the Statistical Hurricane Intensity Prediction Scheme (SHIPS; ?, ?). Within this dataset, maximum potential intensity is calculated following the definition in ? (?). Deep layer shear magnitude is averaged over the TC from $200-800 \mathrm{~km}$ radius.

\subsection{Methodology}

The convective morphology of all images for TCs globally between 2012 and 2016 is analysed, for a total for 3881 individual classifications. These five years are chosen as they correspond to the launch of the AMSR-2 instrument in 2012 and the end of availability of SHIPS data in 2016. Each TC is classified manually using an objective classification scheme. A classification consists of three steps: SE identification, SBC identification and rainband identification. This scheme is manually implemented using a matlab pipeline. The analyst is sequentially shown each image, and prompted to classify it 


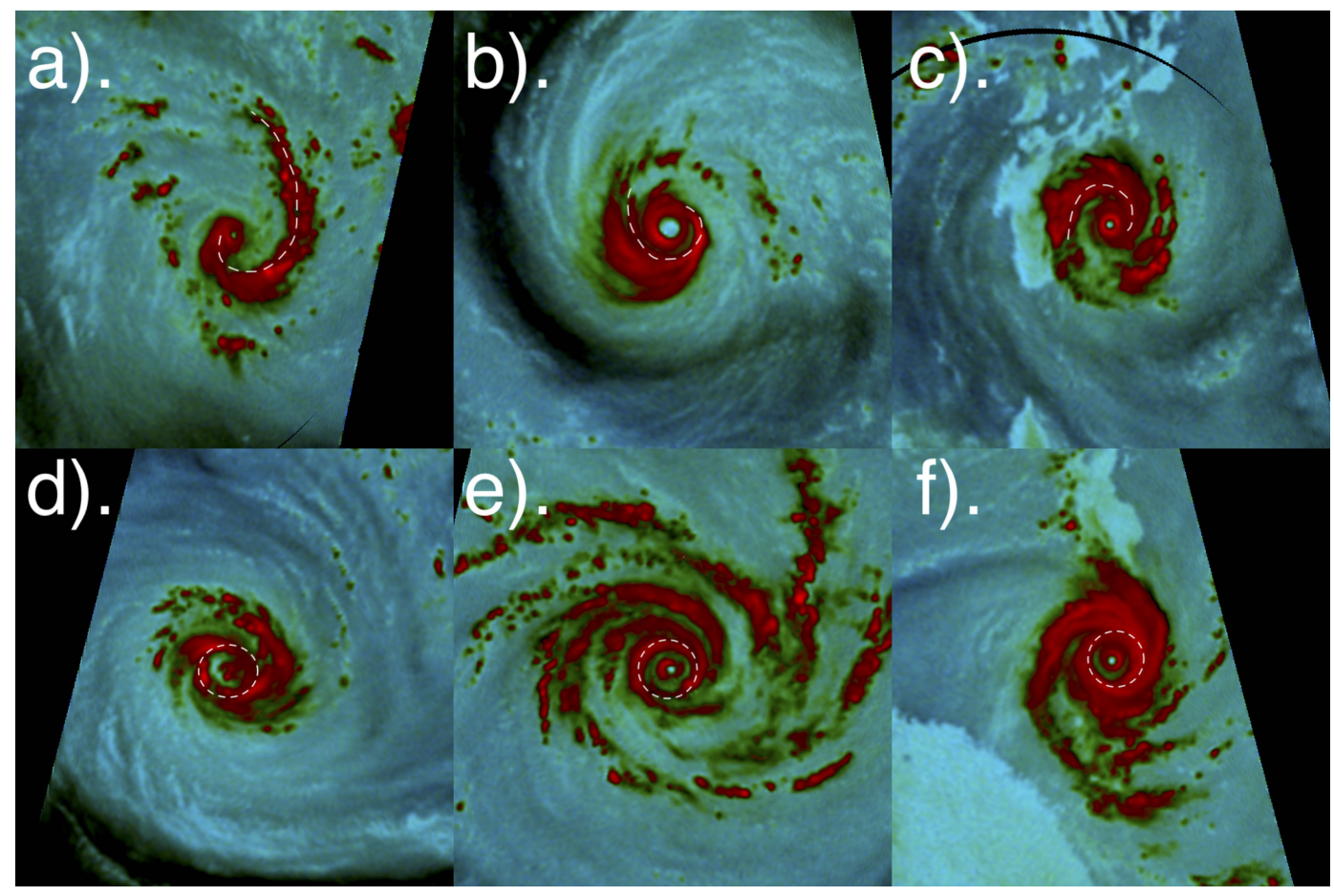

Figure 2. Examples of (a,b,c) SBCs and (d,e,f) SEs identified in 89GHz PMW imagery, for (a) TC Amanda at 01:29Z May 26. 2014, (b) TC Bruce at 23:34Z Dec. 21, 2013, (c) Typhoon Noul 22:15Z May 9, 2015, (d) Typhoon Kilo 7:21Z Sep. 5, 2015, (e) TC Atsani 7:45Z Aug. 18, 2015, (f) Typhoon Meranti 20:26Z Sep. 13, 2016. White dashed lines on images indicate the location of the SBC or SE. 
as $\mathrm{SE} / \mathrm{SBC}$ based on the criteria outlined below. For SBC cases, the extent of the rainband is manually identified on the image to allow the geometry to be quantified.

\subsubsection{Eyewall classification}

The TC is classified as either containing a secondary eyewall (SE) or not containing a secondary eyewall (NSE). A secondary eyewall is defined as a quasi-circular ring of convection with $P C T_{89}<255 \mathrm{~K}$ around the inner eyewall that is at least $2 / 3 \mathrm{com}$ plete with a clear moat. Although these thresholds are somewhat arbitrary, this definition is similar to that used in previous PMW climatologies of SEF (?, ?, ?, ?, ?, ?). Examples of images classified as SE are shown in Figure 2 (d,e,f). Although recent work has used fully automated techniques to identify SEs (?, ?), extensive testing demonstrated that brightness temperature averaging missed large numbers of secondary eyewalls in smaller and asymmetric systems, and was therefore deemed unsuitable for this study.

\subsubsection{SBC classification}

Each image is classified as either containing an SBC (SBC) or not containing an SBC (NoSBC). The seminal work of Willoughby et al. (1984) defined an SBC as 'a group prominent spiral rainbands that maintains a fixed position relative to the vortex, usually on the east side.' As part of this study, multiple classification techniques were trialled using computer vision methods to identify the SBC, however these proved unsuitable given the wide range of TC sizes, SBC presentations and interactions with other elements of the convective structure. A series of criteria are therefore chosen to objectively identify regions of convection corresponding to an SBC as defined by Willoughby et al. (1984).

For the purposes of this study, an SBC is defined as a wavenumber one convective asymmetry with $\mathrm{PC}_{89}<255 \mathrm{~K}$ that satisfies the following four criteria:

1. The SBC has a length to width ratio greater than $2: 1$ and spiral geometry. Here the width of a rainband is defined as the distance across the widest point of the principal band, and the length the distance from the upwind to downwind end of the band.

2. The SBC is separated from the inner core by a clear moat along at least $2 / 3$ of the band length.

3. The structure of the SBC consists of one principal band which all other bands are attached to.

4. The SBC is either attached to the inner core convection surrounding the eyewall or separated by a narrow convection free moat.

Criteria one and two exclude cases where asymmetric convection is observed that does not have a clear spiral structure and is not separated from the inner core. Criterion three is necessary to exclude groups of rainbands without a dominant principal band. Finally, criterion four excludes prominent outer convective complexes which may meet 
the asymmetry and size criteria but are distinct from the SBCs documented in previous observational work (?, ?, ?, ?).

Examples of images classified as SBC are shown in Figure $2(\mathrm{a}, \mathrm{b}, \mathrm{c})$.

\subsubsection{Geometry classification}

For those TCs classified as containing an SBC, the innermost edge of the SBC that is clearly separated from the eyewall is manually identified using a command line tool implemented in MATLAB. Examples of SBCs identified in the PMW dataset are indicated by white dashed lines in Figure $2(\mathrm{a}, \mathrm{b}, \mathrm{c})$. The geometry of the band is then quantified by fitting a logarithmic spiral to the manually identified points using nonlinear least squares minimisation.

A logarithmic spiral is described by the equation

$$
r=a \times e^{b \theta}
$$

where $\mathrm{a}$ and $\mathrm{b}$ are real constants. Although more sophisticated models have been developed to describe TC rainband geometry $(?, ?, ?)$, a logarithmic spiral provides the best fit to the widest variety of rainband types (?, ?), and has been used to model rainbands in recent work $(?, ?)$.

From the logarithmic spiral fit, two parameters are used to characterise each band: the azimuthal extent $(\Psi)$ and crossing angle $(\aleph)$. The azimuthal extent is defined by

$$
\Psi=\left(\theta_{f}-\theta_{i}\right)+2 \pi \times k
$$

with the sign reversed for Southern Hemisphere TCs, where $\theta_{i}$ and $\theta_{f}$ are the start and finish coordinates of the rainband, and $\mathrm{k}$ is the number of complete revolutions of the band around the TC. The crossing angle is defined by

$$
\aleph=\tan ^{-1}\left(\frac{1}{b}\right)
$$

with the sign reversed for Southern Hemisphere TCs. This provides a measure of the circularity of the band, where $0^{\circ}$ is circular and $90^{\circ}$ is a radial straight line. In the context of SEF, these parameters capture the extent to which vorticity and diabatic heating project onto the azimuthal mean, and the degree to which the band has axisymmetrised as it evolves into a SE. These parameters are preferred over using the absolute length of the band as they are independent of the absolute vortex size.

\subsubsection{Kernel density estimation}

Kernel density estimation (KDE) is used to compare the joint distributions of rainband crossing angle and azimuthal extent for TCs in different basins and at different stages prior to SEF. This method provides an estimate of the probability distribution of a random variable (?, ?), and is here used with a Gaussian kernel and implemented using the python seaborn library. 

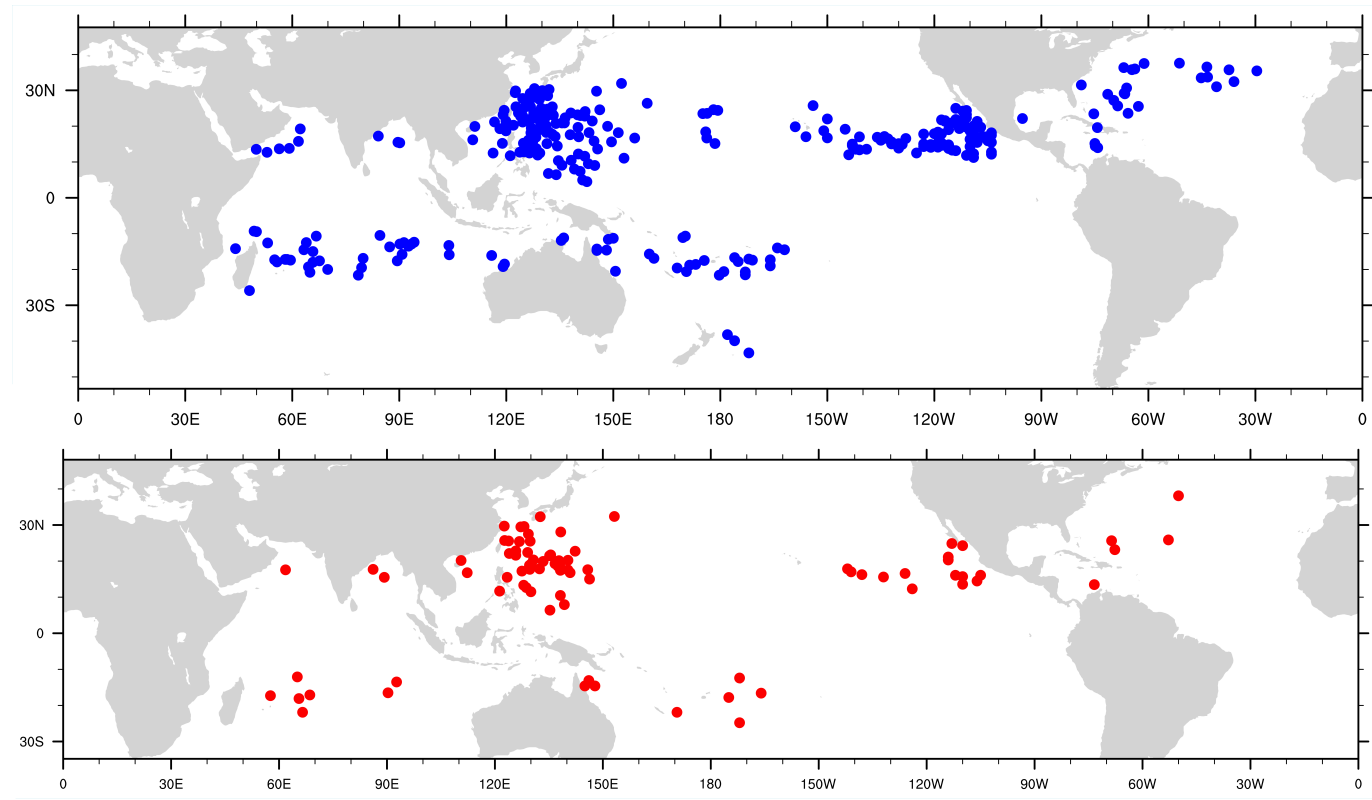

Figure 3. Geographic distributions of events included in the sample for (top) SBCF events and (bottom) SEF events.

\section{Climatology of stationary banding complex formation}

To avoid uncertainty in the timing of stationary banding complex formation (SBCF) events due to the irregular temporal resolution of the PMW dataset, an SBCF event is defined as a sequence of two PMW images within 6 hours where the first is classified as NoSBC and the second as SBC. The second image in the sequence, i.e the first image classified as SBCF is then classified as an SBCF datapoint. Similarly, a NoSBCF event is defined as a sequence of two PMW images within 6 hours where neither image is classified as containing an SBC. Here the NoSBCF event point is taken as the second image. Using these definitions, a total of 318 SBCF and 1730 NoSBCF events are included in the dataset.

Geographic locations of SBCF events are shown in Figure 3 (a). A total of 1506 PMW images contain an SBC, comprising $39 \%$ of all PMW overpasses. In this analysis, differences in morphology are considered by basin for the North Atlantic, Eastern Pacific, Western Pacific, South Indian Ocean and South Pacific. As only 93 PMW overpasses are included in the North Indian Ocean, this basin is not included. Percentages of passes with an SBC and total sample numbers for each basin are summarised in Table 1. The overall incidence of passes with an SBC does not vary significantly between basins, ranging from $42 \%$ in the Western Pacific basin to $35 \%$ in the Southern Indian Ocean. There is a small but significant difference in event latitudes, with a median of 18.17 degrees for SBCF and 19.58 degrees for NoSBCF, although the physical reasons for this difference is not known. 
Table 1. SBC statistics by basin, where $\aleph$ is the crossing angle and $\Psi$ is the azimuthal extent.

\begin{tabular}{ccccc}
\hline Basin & Total images & $\%$ SBC & Median & Median $\Psi$ \\
\hline Western Pacific & 1554 & $42 \%$ & $10.42^{\circ}$ & $1.48 \pi \mathrm{rad}$ \\
Eastern Pacific & 897 & $37 \%$ & $12.24^{\circ}$ & $1.29 \pi \mathrm{rad}$ \\
South Indian Ocean & 492 & $35 \%$ & $12.19^{\circ}$ & $1.31 \pi \mathrm{rad}$ \\
South Pacific & 355 & $39 \%$ & $13.88^{\circ}$ & $1.26 \pi \mathrm{rad}$ \\
North Atlantic & 490 & $36 \%$ & $12.98^{\circ}$ & $1.21 \pi \mathrm{rad}$ \\
\hline Total sample & 3881 & $39 \%$ & $11.54^{\circ}$ & $1.37 \pi \mathrm{rad}$ \\
\hline
\end{tabular}

In order to assess the role of the large scale environmental conditions in SBCF, the intensity, maximum potential intensity and deep layer shear distributions are compared for the SBCF and NoSBCF events. Differences between the two groups are significant at the $95 \%$ level for all parameters using a 2 sample Kolmogorov-Smirnov (KS) test (Figure 4). SBCF events occur in an environment with higher median intensity (85kts compared with $81.82 \mathrm{kts}$ ), higher median maximum potential intensity (133.65kts compared to $124.99 \mathrm{kts}$ ), and lower median deep layer shear (11.17kts compared to $12.72 \mathrm{kts}$ ).

\section{Geometry of SBCs}

SBC geometry is analysed with respect to the crossing angle and azimuthal extent parameters. Figure 5 shows the distribution of crossing angles for all SBCs in the sample. Crossing angles range from a minimum of 0.19 degrees to a maximum of 61.78 degrees, with a median of 11.54 degrees. Examples of the extremes of the distribution are shown in Figure $5(\mathrm{~b}, \mathrm{c})$.

Figure 6 shows the variation in crossing angle and azimuthal extent for PMW overpasses in different basins. TCs in the Western Pacific basin are significantly more likely to have lower crossing angle, with a median crossing angle of 10.42 degrees compared to 12.24, 13.88, 12.19 and 12.98 degrees for the Eastern Pacific, South Pacific, South Indian Ocean and North Atlantic basins respectively. The crossing angle of an SBC varies depending on the current intensity, maximum potential intensity and deep layer shear magnitude. Differences in crossing angle for SBCs in different environmental conditions are analysed by comparing geometry of rainbands in the first and fourth quartiles for each of these three parameters (Figure 8). Differences in crossing angle are significant at the $99 \%$ level for maximum intensity and deep layer shear magnitude using a 2 sample KS test. Higher maximum intensity occurs with lower crossing angle, with a median of 13.61 degrees (10.28 degrees) for values in the first (fourth) quartile. Lower crossing angle is also associated with lower shear, with a median of 10.40 degrees (13.08 degrees) for values in the first (fourth) quartile respectively. 

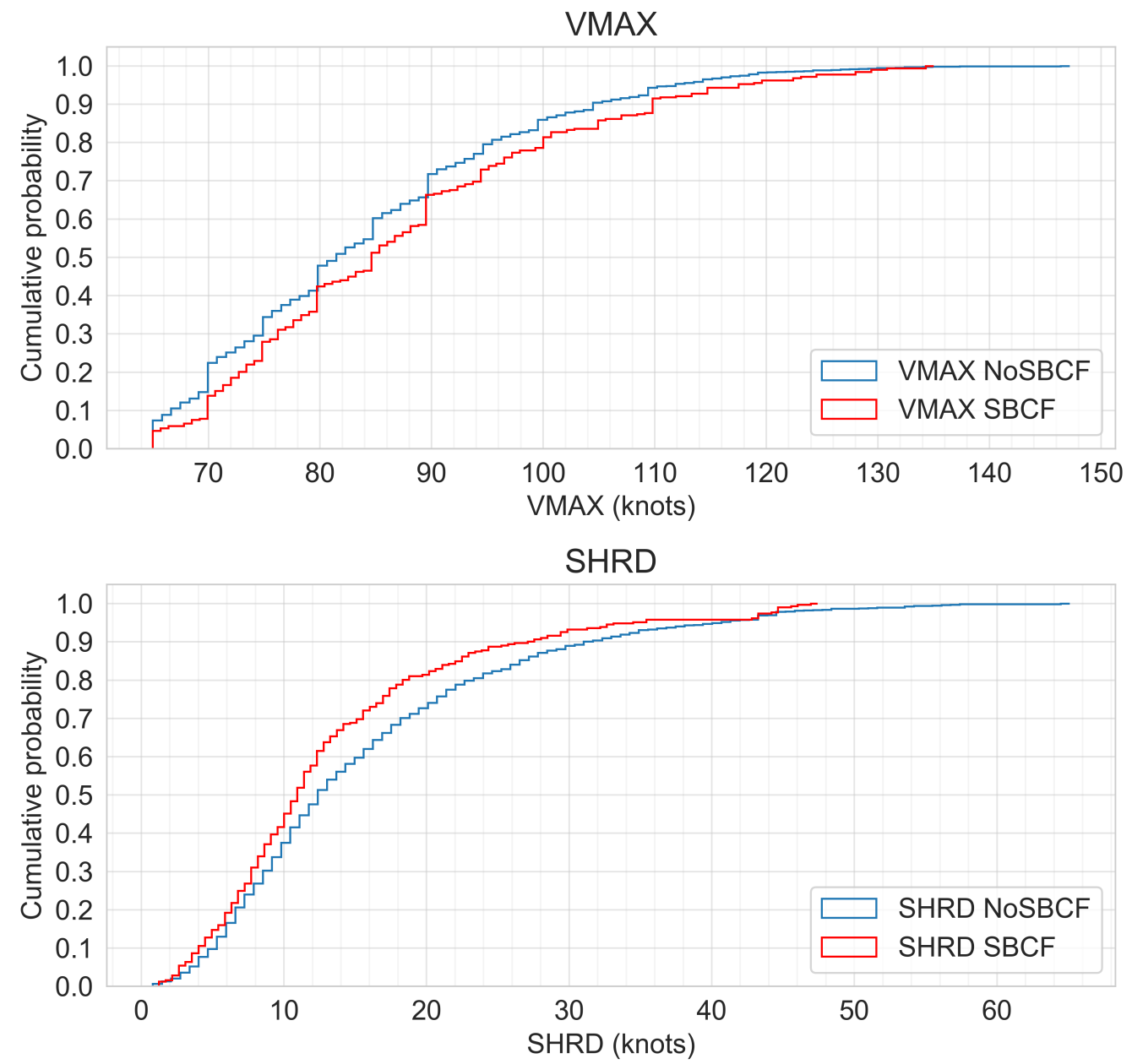

VMPI

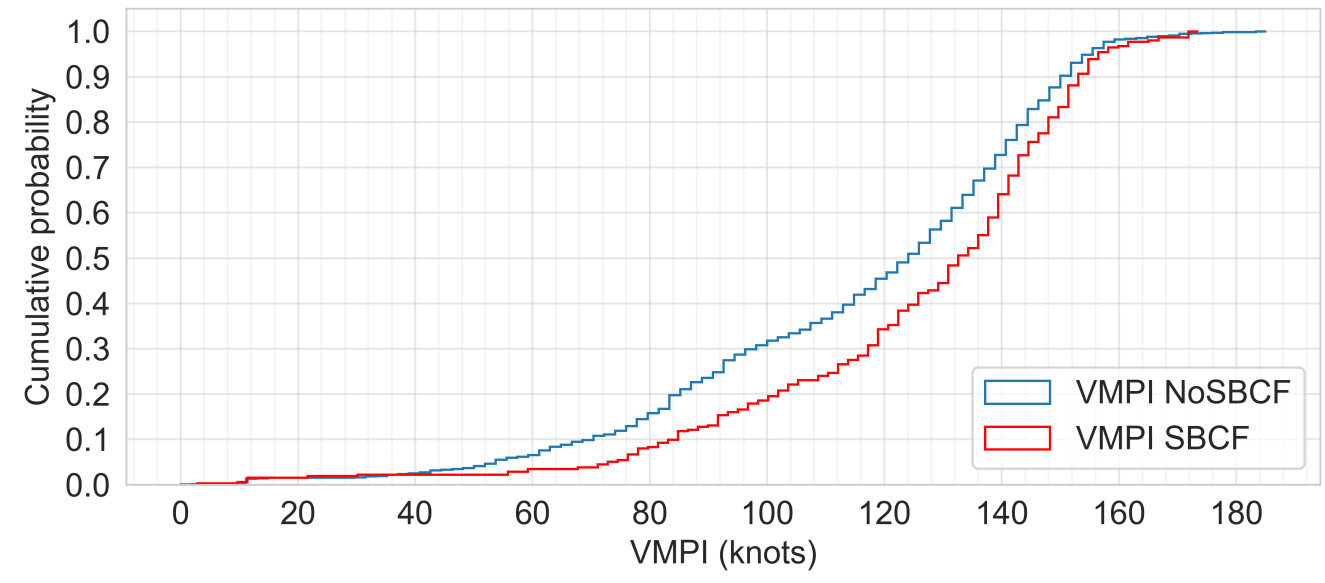

Figure 4. Cumulative distributions comparing environmental conditions for the SBCF and NoSBCF groups, maximum intensity (top), deep layer shear (middle) and maximum potential intensity (bottom).

This article is protected by copyright. All rights reserved. 

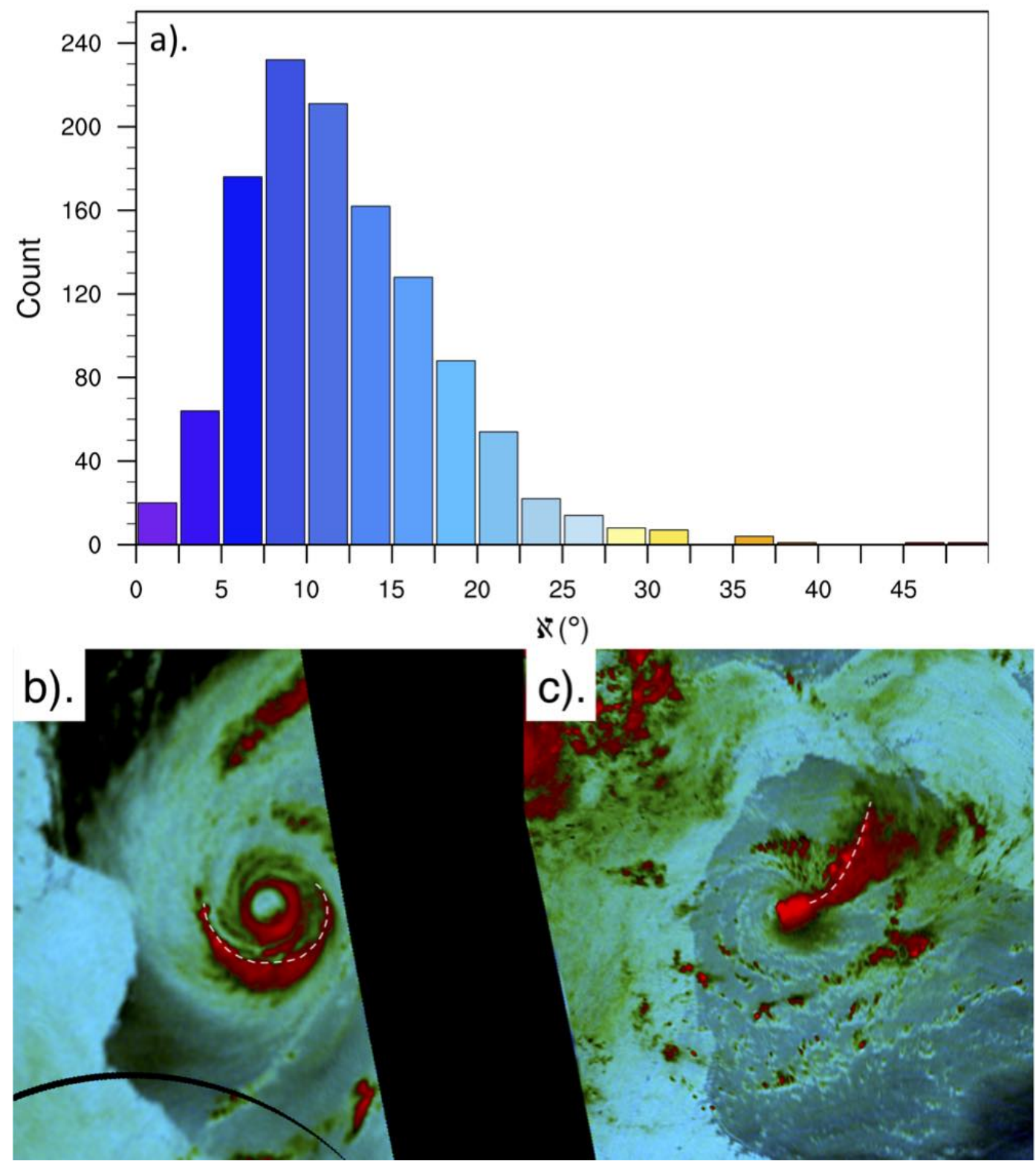

Figure 5. Crossing angle variation between SBCs, showing (a) crossing angle distribution for all bands in the sample, (b) example of an SBC with crossing angle of 0.89 degrees in TC Funso at 05:43Z Jan. 26, 2012. (c) Example of an SBC with crossing angle of 61.78 degrees in Hurricane Ingrid at 07:59Z Sep. 15, 2013. 

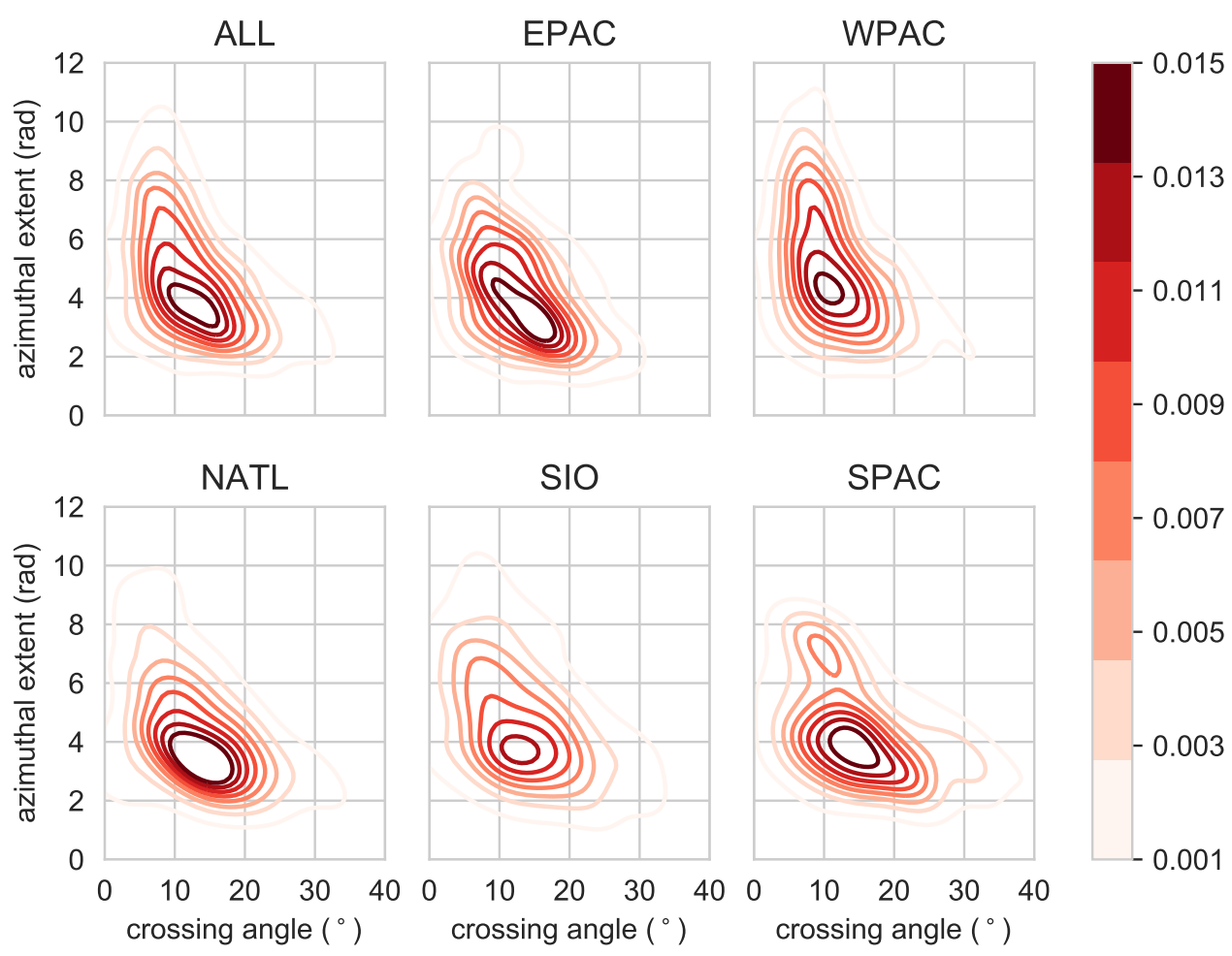

0.007

$-0.005$

$-0.003$

0.001

Figure 6. KDE plots showing variations in SBC geometry by basin. (a) Total sample (ALL), (b) Eastern Pacific (EPAC), (c) Western Pacific (WPAC), (d) North Atlantic (NATL), (e) South Indian Ocean (SIO), (f) South Pacific (SPAC). 

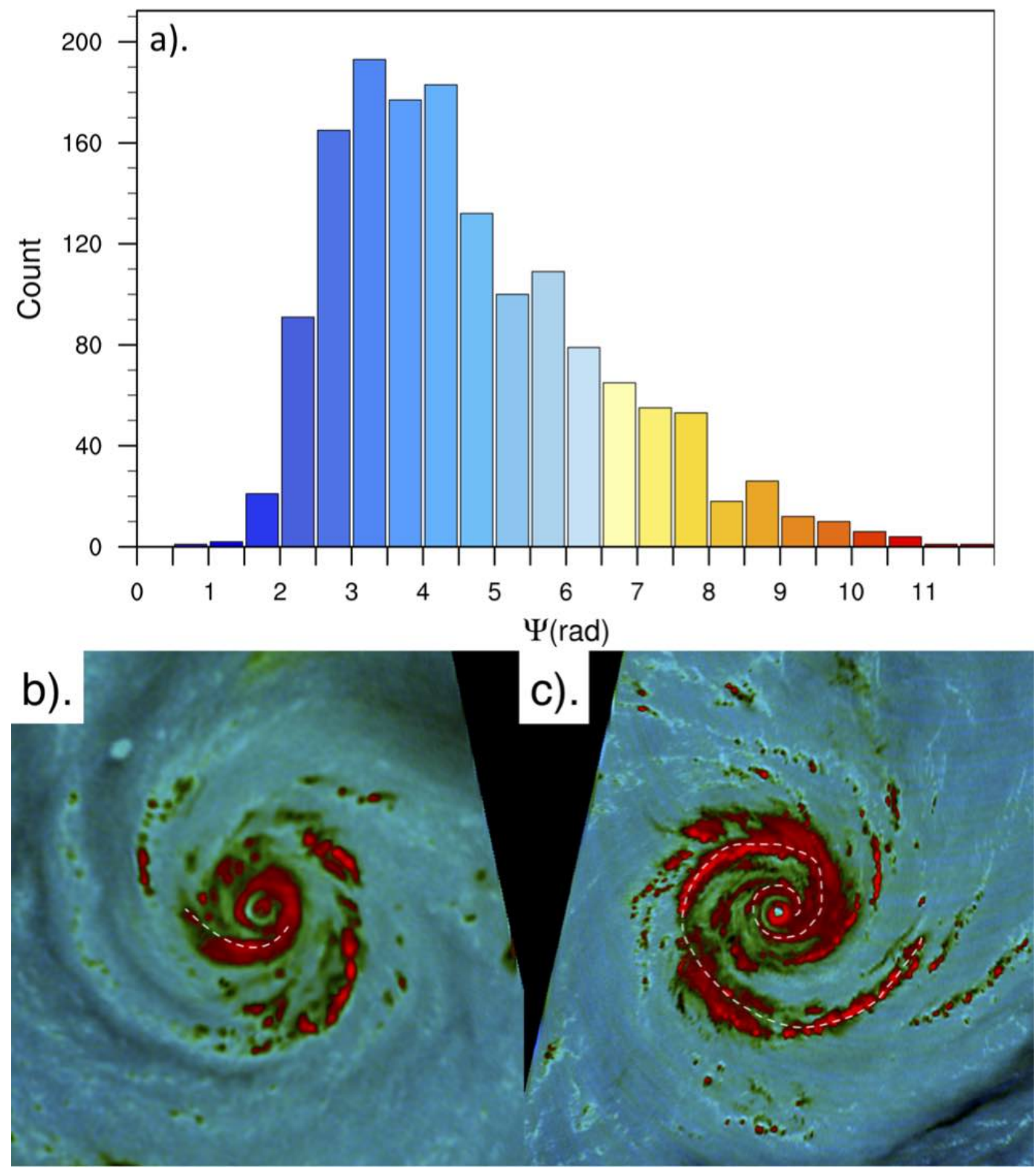

Figure 7. Azimuthal extent variation between SBCs, showing (a) azimuthal extent distribution for all bands in the sample, (b) example of an SBC with azimuthal extent of $0.62 \pi$ radians in TC Giovanna at 04:08Z Feb. 11, 2012. (c) Example of an SBC with azimuthal extent of $4.02 \pi$ radians in Typhoon Nangka at 03:27Z Jul. 9, 2015. 
The azimuthal extent distribution for all SBCs in the sample is shown in Figure 7. Values range from $0.29 \pi$ to $4.02 \pi$ radians, with a median of $1.37 \pi$ radians. Examples of the extremes of the distribution are shown in Figure 7 (b, c). As for the crossing angle, differences in azimuthal extent are apparent between basins and in different large scale environmental conditions. Azimuthal extents are significantly higher in the Western Pacific with a median of $1.48 \pi$ radians compared to $1.29 \pi, 1.26 \pi, 1.31 \pi$ and $1.21 \pi$ radians for the Eastern Pacific, South Pacific, South Indian Ocean and North Atlantic basins respectively (Figure 6).

Analysing the differences between environmental conditions in the same manner as the crossing angle, significant differences in azimuthal extent are observed for SBCs in the first compared to fourth quartile of each of the three parameters (Figure 8). Higher maximum intensity and maximum potential intensity occur with significantly higher azimuthal extent, with first (fourth) quartile median values of $1.17 \pi$ radians ( $1.60 \pi$ radians) and $1.22 \pi$ radians ( $1.38 \pi$ radians) for maximum intensity and maximum potential intensity respectively. Lower magnitude of deep layer shear is associated with higher azimuthal extent, with median values of $1.50 \pi$ radians (1.21 $\pi$ radians) for SBCs in the first (fourth) quartile. Note that higher intensity is associated with lower crossing angle, as expected, but this relationship is opposite for VMPI. We speculate that this is because most TCs do not reach their VMPI, due to factors such as wind shear that are not included in the formulation of potential intensity. For dynamical reasons, crossing angle should be more physically related to actual rather than potential intensity.

\section{Morphology prior to secondary eyewall formation}

A total of 147 unique SEF events are observed in the sample. As for SBCF events, to ensure that the time of SEF is accurate to within $6 \mathrm{hrs}$ a SEF event is defined as a sequence of two PMW overpasses within 6 hours of each other classified as NSE and SE respectively. For comparison, a No SEF event is defined as a PMW image where no subsequent image contains a SE within 24 hours. Applying this criterion, 84 SEF events and 3078 NoSEF events are included in the dataset. Locations of the SEF events are shown in Figure 3 (b).

Given the discontinuous nature of the $89 \mathrm{GHz}$ PMW coverage, it is challenging to track the evolution of rainbands leading up to SEF with the current dataset. Images are therefore binned in different time periods prior to SEF to investigate the average structure of the TC at intervals prior to SEF. PMW overpasses within $24 \mathrm{hrs}$ of the SEF events described above are divided into four groups: 0-6hrs, $6-12 \mathrm{hrs}, 12-18 \mathrm{hrs}$ and $18-24 \mathrm{hrs}$ prior to SEF. Statistics of sample size and morphology for images in each of these four time periods are shown in Table 2. SBCs are observed in 79\%, 55\%, $60 \%$ and $72 \%$ of events in 0-6 hours, 6-12 hours, 12-18 hours and 18-24 hours respectively, compared to $35 \%$ in the No SEF group. In the 24 hours prior to SEF, a TC is therefore substantially more likely to be classified as SBC. The increase in percentage of events with an SBC at 18- 

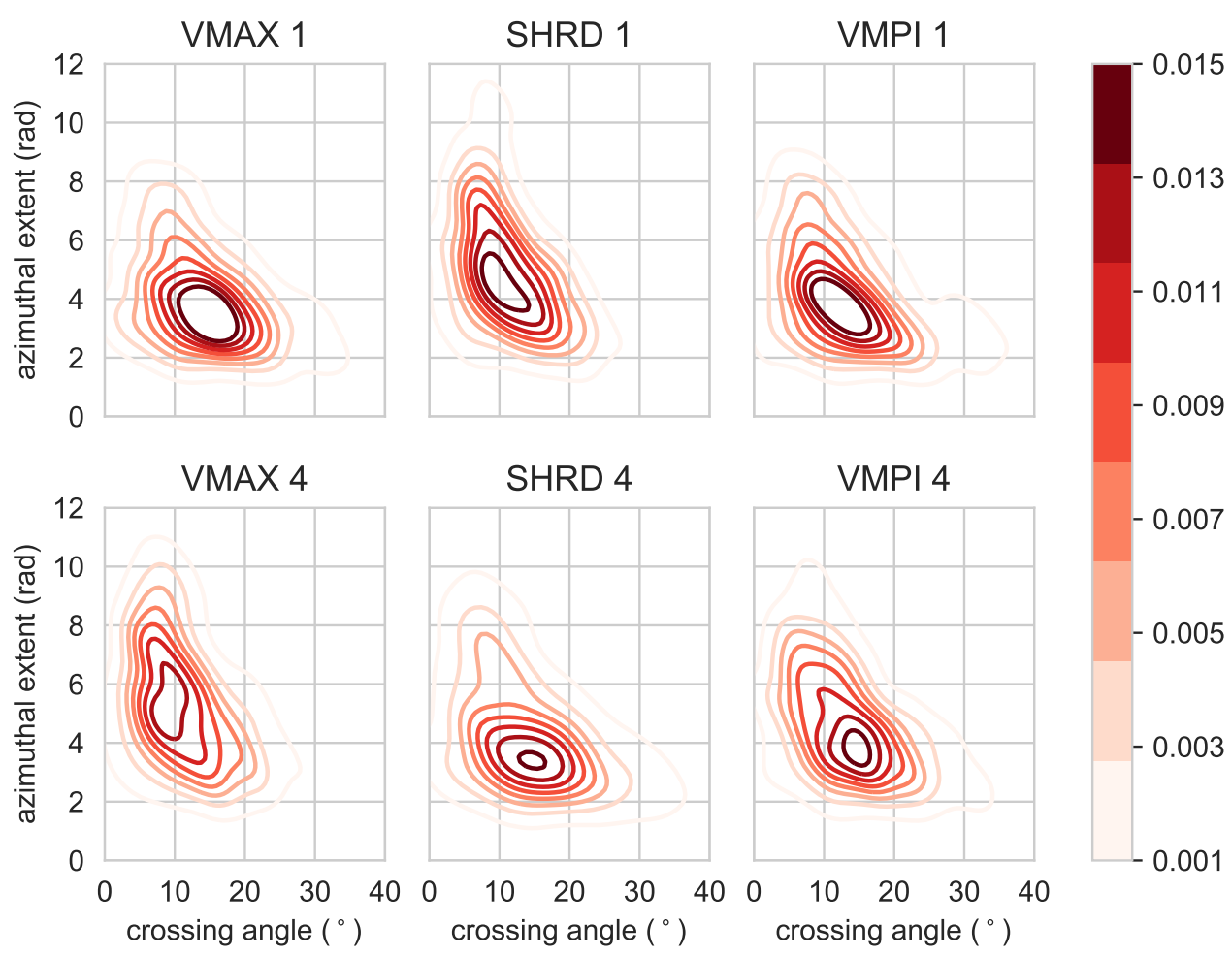

$-0.007$

$-0.005$

$-0.003$

0.001

Figure 8. KDE plots demonstrating variations in SBC geometry in different environmental conditions. First quartile of intensity (VMAX 1), fourth quartile of intensity (VMAX 4), first quartile of deep layer shear (SHRD 1), fourth quartile of deep layer shear (SHRD 4), first quartile of maximum potential intensity (VMPI 1) and fourth quartile of maximum potential intensity (VMPI 4). 

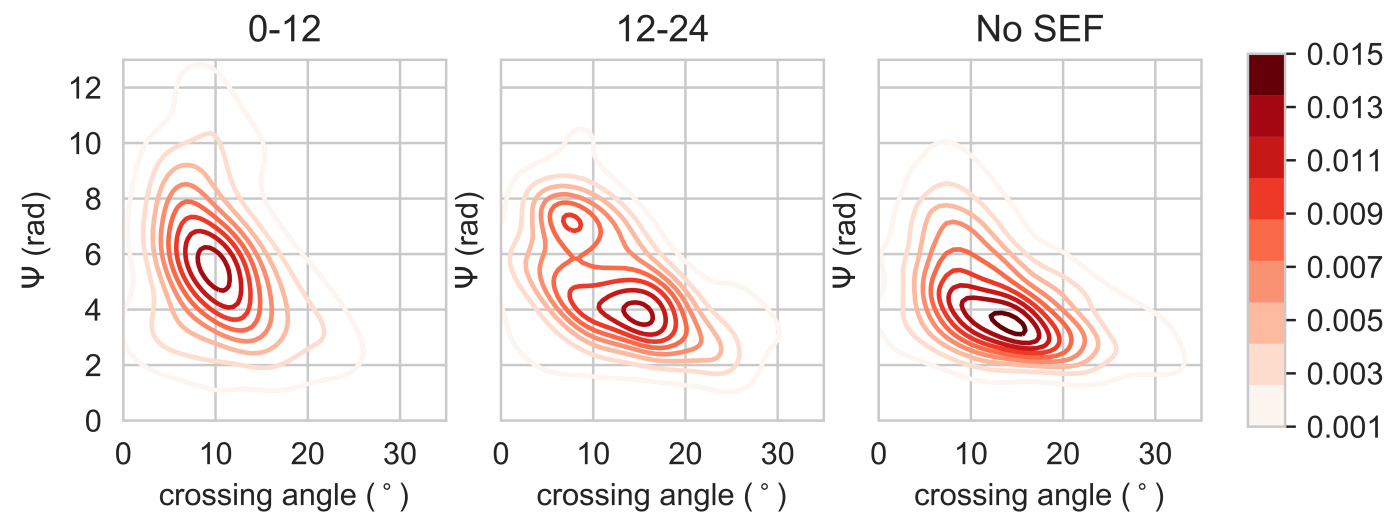

Figure 9. KDE plots of variation in SBC geometry at different time periods prior to SEF, from left to right: 0-12 hours prior to SEF, 12-24 hours prior to SEF, cases with no SEF within 24 hours.

24 hours is partially explained by TCs in the sample with multiple SEF events where data points are counted in both the 0-6 hours and 18-24 hours groups.

The geometry of SBCs varies significantly from the sample median prior to SEF. KDE plots of band geometry at 0-12 hours, 12-24 hours and cases where no SEF event occurs within 24 hours are shown in Figure 9. Differences between all distributions are significant at the $99 \%$ level using a 2D-Kolmogorov Smirnov test. SBCs observed within 12 hours of SEF have significantly higher azimuthal extent and lower crossing angle than those with no SEF within 24 hours, with median values of 9.89 degrees and $1.69 \pi$ radians respectively. This compares to azimuthal extents and crossing angle medians of 11.67 and 4.5 for the 12-24 hour group, and 12.14 degrees and $1.30 \pi$ radians for the noSEF group. The distribution at 12-24 hours is bimodal, suggesting that some TCs may maintain a coherent SBC configuration with high azimuthal extent and low crossing angle for longer than others prior to SEF.

An observation from Figure 9 is that the No SEF group contains cases that have high azimuthal extent and low crossing angle but do not go on to form secondary eyewalls. In the context of previously proposed hypotheses that SEF is triggered when rainband heating and vorticity project substantially onto the azimuthal mean (?, ?, ?), it would be expected that these rainbands would develop into secondary eyewalls. To assess why SEF does not eventuate in these cases, the maximum intensity, potential intensity and deep layer shear magnitude are compared for images in the 0-6 hour group, regardless of SBC morphology, and those in the No SEF group with crossing angle (azimuthal extent) less than (greater than) the 50th percentile of values in the 0-6 hour group. Histograms of the distributions for the two groups for intensity, maximum potential intensity and deep layer shear are shown in Figure 10. Differences between distributions are modest, however are significant at the $90 \%$ level using a 2-sample KS test for all three 
Table 2. SEF statistics by time period.

\begin{tabular}{cccccc}
\hline Time period & Total SEF events & Total images & \% events SBC & Median $\left(^{\circ}\right)$ & Median $\Psi(\mathrm{rad})$ \\
\hline $0-6 \mathrm{hr}$ & 84 & 205 & $79 \%$ & 9.72 & $1.68 \pi$ \\
$6-12 \mathrm{hr}$ & 55 & 102 & $55 \%$ & 11.18 & $1.79 \pi$ \\
$12-18 \mathrm{hr}$ & 62 & 105 & $60 \%$ & 10.33 & $1.45 \pi$ \\
$18-24 \mathrm{hr}$ & 60 & 120 & $72 \%$ & 12.37 & $1.40 \pi$ \\
\hline No SEF & - & 3078 & $35 \%$ & 12.14 & $1.30 \pi$ \\
\hline
\end{tabular}

parameters. Cases with no SEF have lower median shear and intensity and higher maximum potential intensity.

\section{Discussion and conclusion}

Using $89 \mathrm{GHz}$ PMW data, we compiled the first climatology of the formation and geometry of the SBC, and used this to quantify changes in convective morphology prior to SEF. Specifically, we have demonstrated that $79 \%$ of SEF events followed the same pathway to SEF, with three stages consisting of:

1. Formation of an SBC.

2. Evolution of the SBC geometry to a configuration with high azimuthal extent and low crossing angle as compared to sample medians.

3. Axisymmetrisation of the SBC into a SE.

Results presented in sections 3-5 provided insight into the climatological characteristics of each stage of this process.

SBC formation is demonstrated to be largely independent of basin, unlike SEF which is considerably more common in the Western Pacific than other basins (e.g Hawkins and Helveston, 2004). SBC formation is more likely in TCs with higher intensity and potential intensity and lower deep layer shear. The limited previous work that has investigated SBCF has suggested that the SBC forms as a result of the asymmetric vortex response to deep layer shear (Willoughby et al., 1984; Riemer and Montgomery, 2011; Riemer, 2016). From this perspective, it would be expected that SBCF is more likely in an environment with stronger VWS. Although storm motion is also a contributing factor to convective asymmetry, this is second order to the effect of vertical shear (?, ?, ?), and is unlikely to play a role in the formation of the SBC $(?, ?)$. This behaviour is not observed in this study. Deep layer shear values are significantly lower for cases in the SBCF group, however SBCF is demonstrated to occur in a wide range of shear magnitudes (Figure 4, bot- 

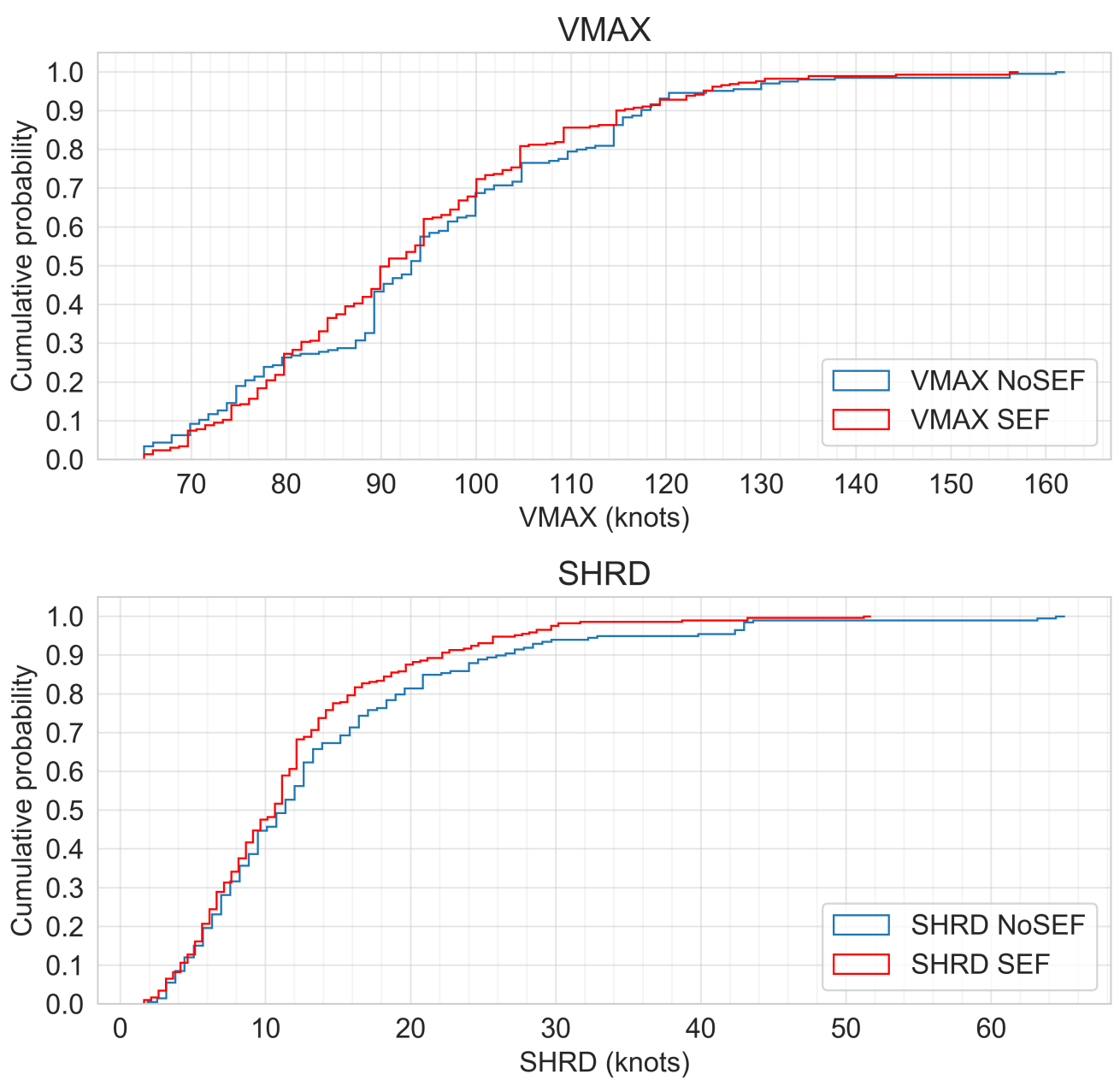

VMPI

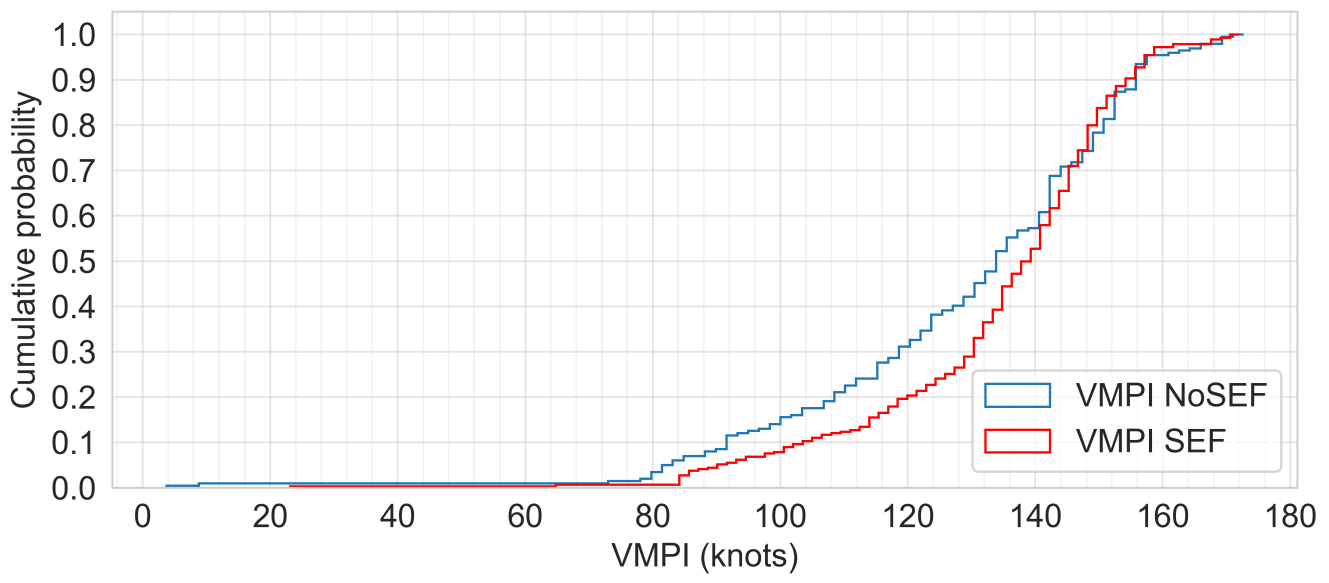

Figure 10. Cumulative distributions comparing environmental conditions for the TCs with an SBC within 6 hours of SEF occurring and those with azimuthal extent greater than the 50th percentile of $0-6 \mathrm{hr}$ sample azimuthal extents and crossing angle less than the 50th percentile osf 0-6hr sample crossing angles, (top) intensity, (middle) deep layer shear and (bottom) maximum potential intensity.

This article is protected by copyright. All rights reserved. 
tom). This suggests that further investigation of SBC formation mechanisms is required using high resolution datasets or numerical simulations.

Considerable variation is observed in the geometry of SBCs. SBCs in TCs with higher intensity in an environment with lower deep layer shear and higher potential intensity have significantly lower crossing angle and higher azimuthal extent. The dynamical reasons for these variations in azimuthal extent can be understood in terms of vortex tilt. A vortex in deep layer shear tilts downshear, inducing a positive low level potential temperature and vorticity anomaly downshear and negative anomalies upshear (?, ?). Higher maximum potential intensity is associated with more favourable storm averaged conditions for deep convection, enhancing the likelihood of convective development on the drier upshear side of the vortex. A vortex in a lower shear environment has reduced tilt, again reducing the dry anomaly upshear and allowing for the SBC to wrap further around the TC. The role of environmental conditions in determining the crossing angle is not immediately clear. The crossing angle of the SBC is likely related to the topology of the low level flow controlling where developing convection is advected within the vortex (?, $?)$.

Our statistical results strongly support the hypothesis that SEF is influenced by the development and morphology of an SBC. This is first time that a preferred convective pathway to SEF has been documented. It is instructive to consider these results in the context of previously proposed hypotheses of the dynamics of SEF. The formation of an SBC within $6 \mathrm{hrs}$ of SEF in $79 \%$ of cases strongly suggests that asymmetric processes are important in SEF, for instance stratiform processes (?, ?) and asymmetric boundary layer inflow forcing $(?, ?)$. The preference for higher azimuthal extent in the 6 hours prior to SEF provides observational evidence that the projection vorticity accumulation and diabatic heating within the rainbands onto the azimuthal mean may contribute to the development of the SE, as previously observed in numerical simulations (?, ?, ?). It is noted, however, that further work is required to explain why many SBCs with high circularity and azimuthal extent do not ultimately evolve into SEs. These results strongly suggest that symmetric processes such as VRWs (?, ?) and beta skirt axisymmetrisation $(?, ?)$ alone are insufficient to explain SEF in these cases, though it is possible that they operate in cases where no SBC is observed or in conjunction with other asymmetric mechanisms. It is important to note that given the nature of this statistical analysis, it is only possible to state that there is a correlation between the SBC and SEF. Further work using numerical simulations would be required to establish whether the SBC causes SEF.

Future work is required to explore changes in morphology in cases where an SBC is not present prior to $\mathrm{SEF}$, and to extend the results presented in this study to a larger dataset. Additional years of data are needed to strengthen the conclusion of this paper and to ensure that the statistical distributions shown here reach full statistical stationarity. A fully automated rainband classification algorithm capable of fitting log spirals to all rainbands as opposed to only SBCs has been developed using a combination of a 
convolutional neural network and unsupervised clustering (?, ?). Though this algorithm currently has low accuracy, it is anticipated that future versions will be applied to further explore morphological evolution prior to SEF. In addition, composites of high resolution Doppler radar data will be used to improve understanding of dynamical processes at each stage of SEF.

\section{Acknowledgments}

This research was partially supported through the funding from the Earth System and Climate Change Hub of the Australian government's National Environmental Science Programme. The analyses were partly performed on the National Computational Infrastructure system, supported by the Australian Government. The authors would also like to thank the Australian Research Council Centre of Excellence for Climate System Science for funding to support this research. Datasets and software used in this research are available from the National Computational Infrastructure (NCI) data catalog at https://geonetwork.nci.org.au/geonetwork/srv/eng/catalog.search.

\section{References}


b).

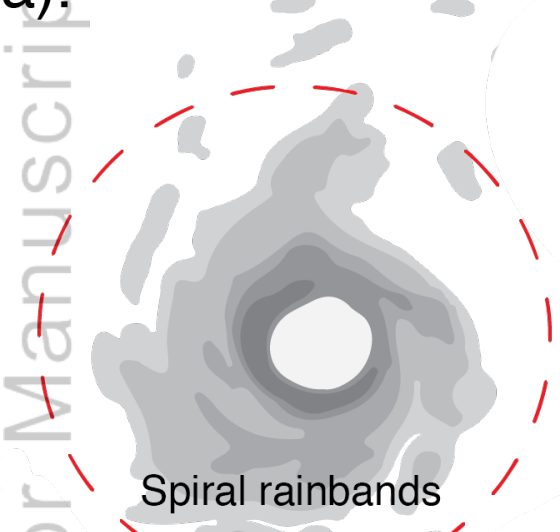

\section{Spiral rainbands}

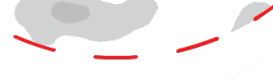

c).

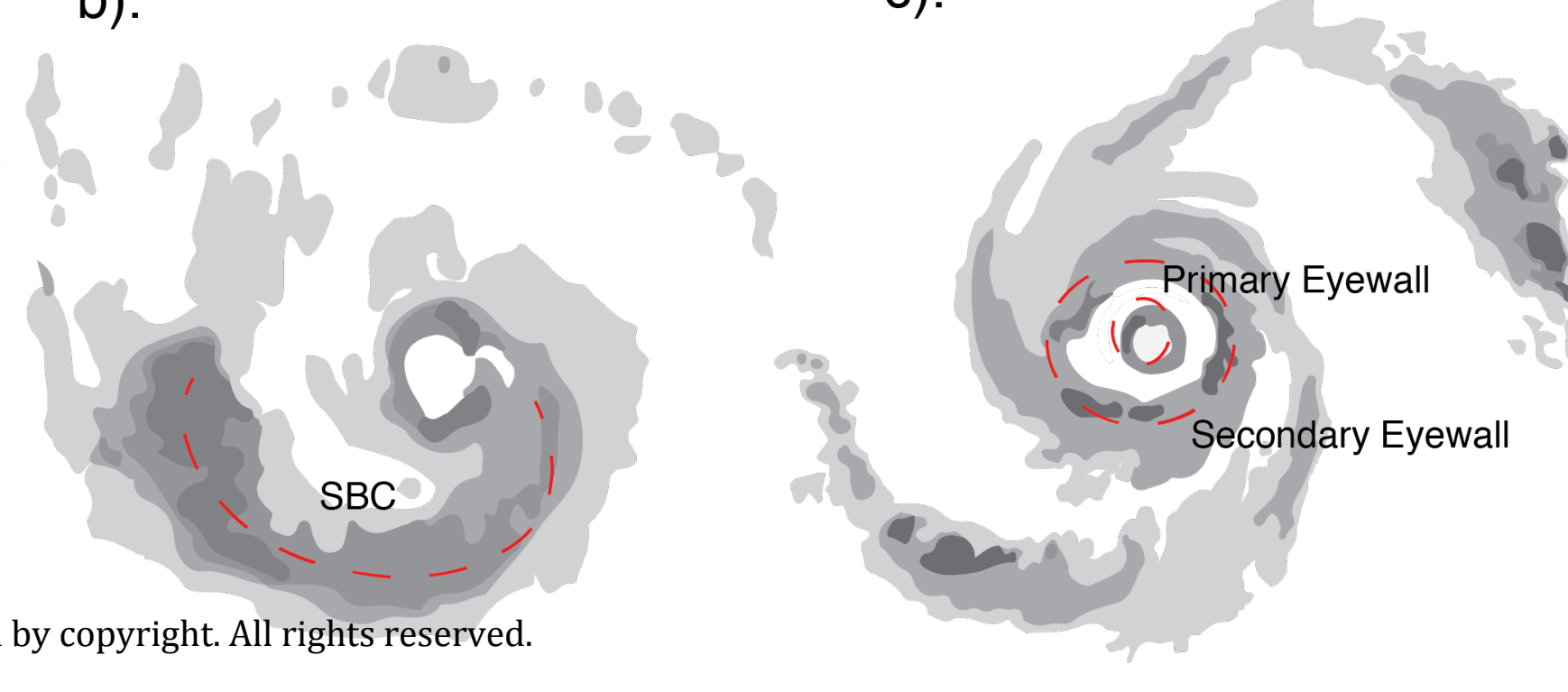


Figure 2.
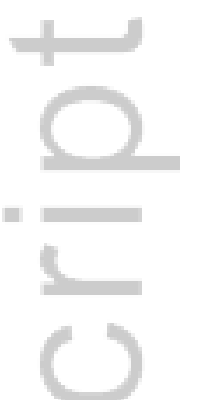

$\infty$
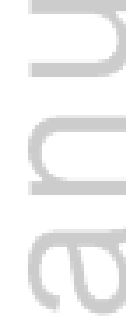

This article is protected by copyright. All rights reserved. 


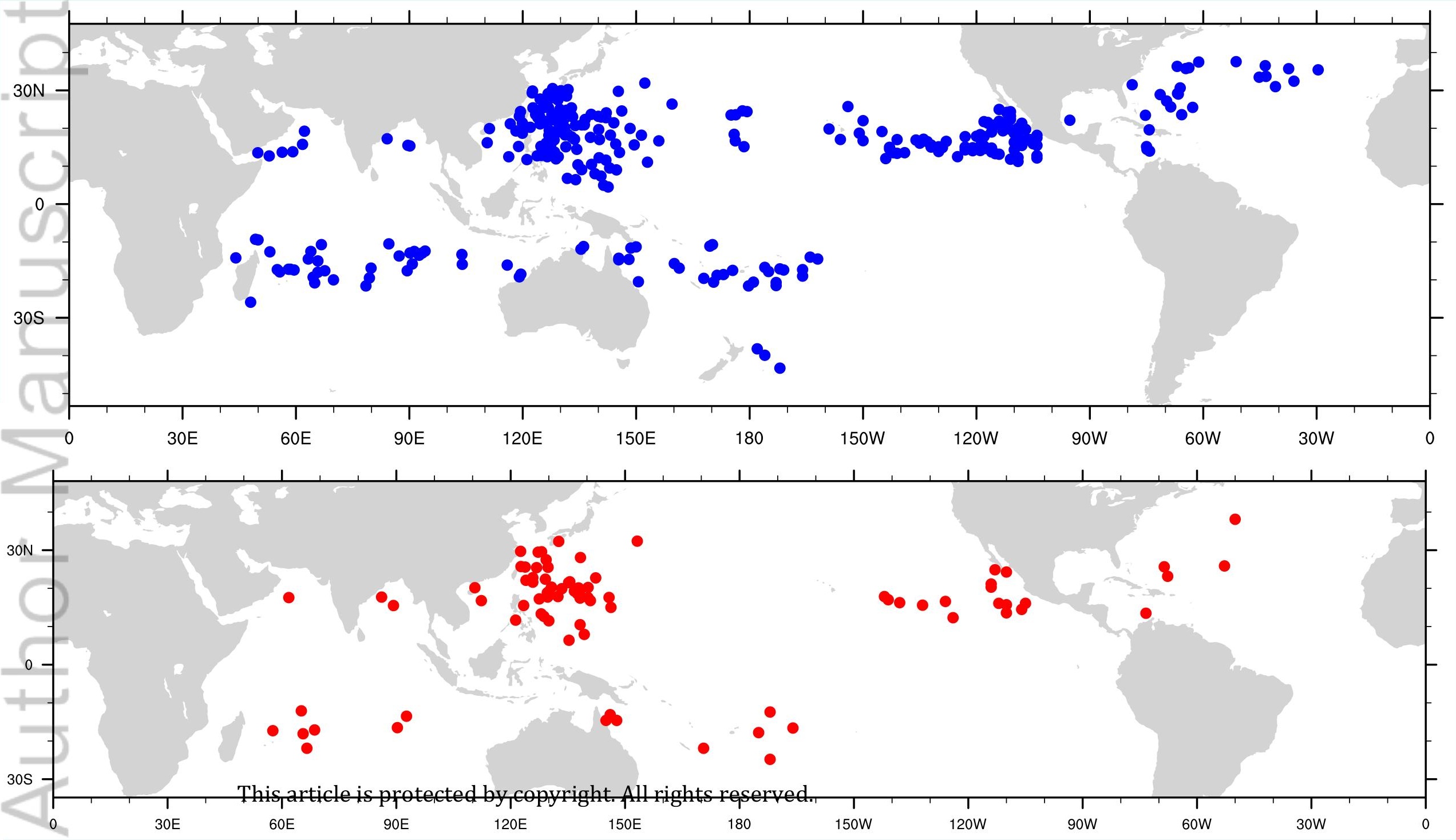


VMAX

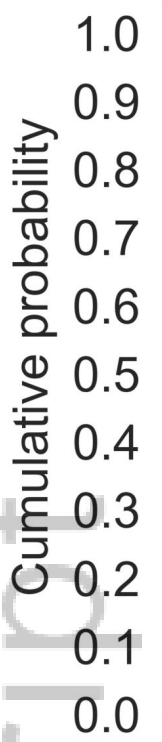

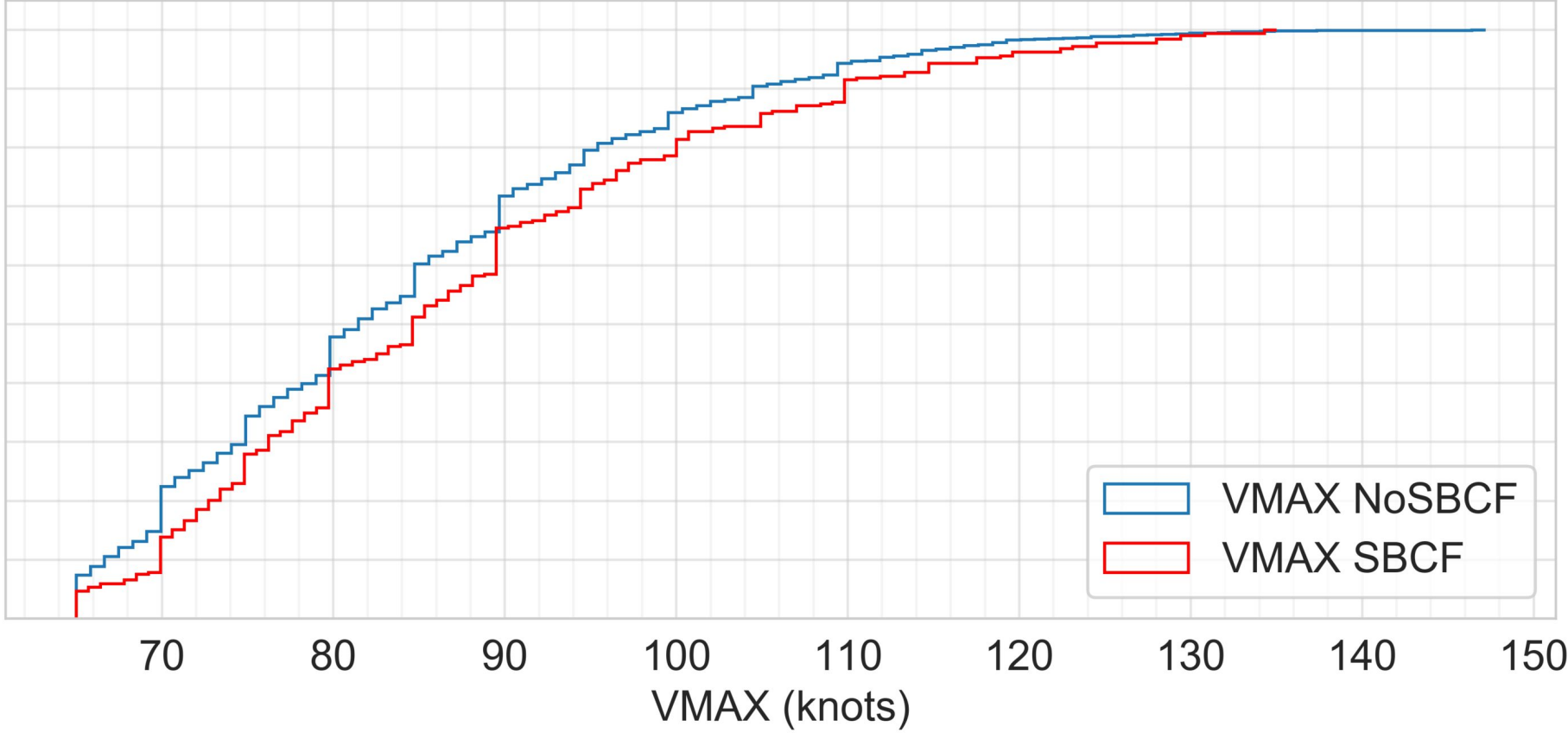

SHRD
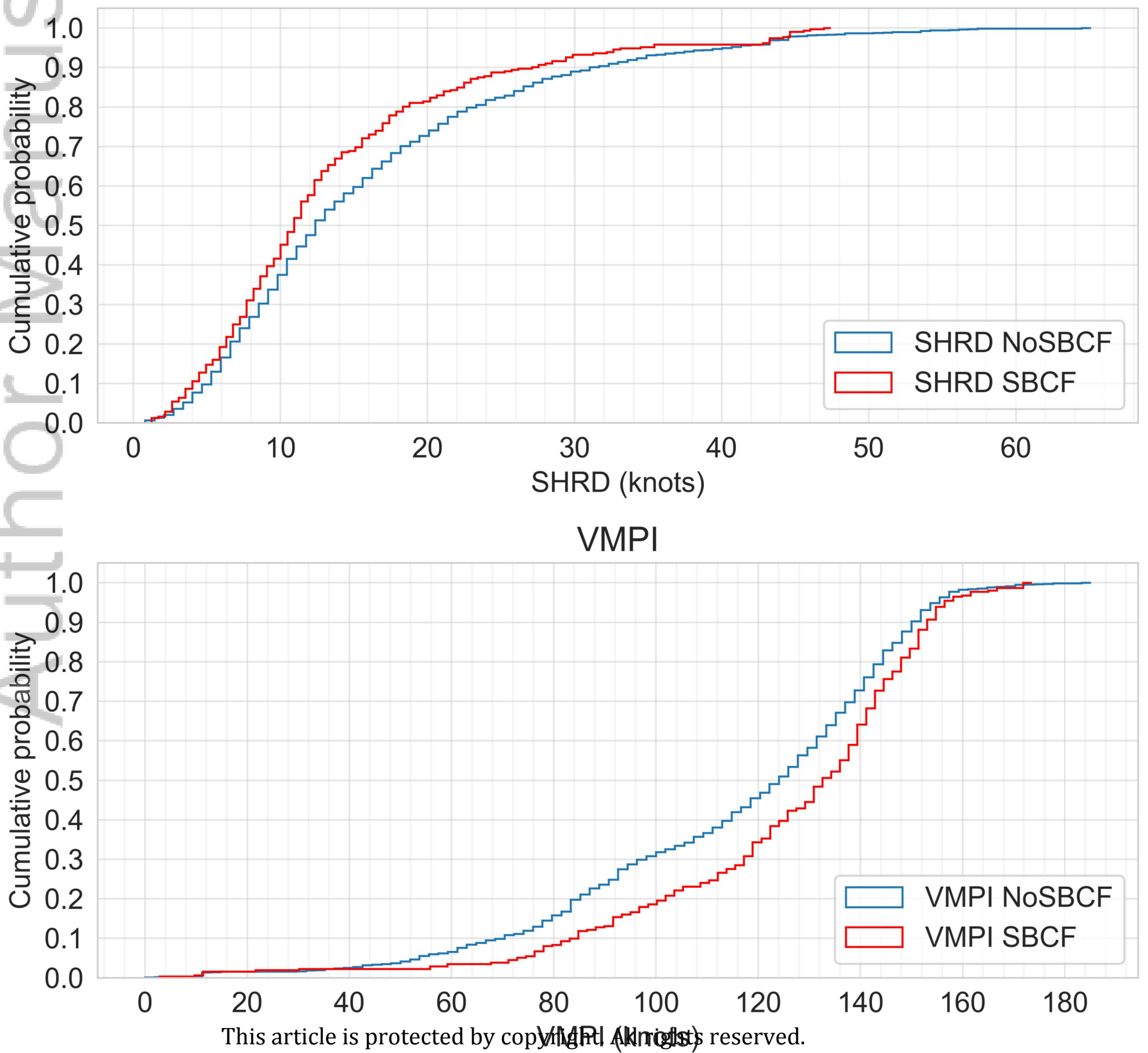
Figure 5.
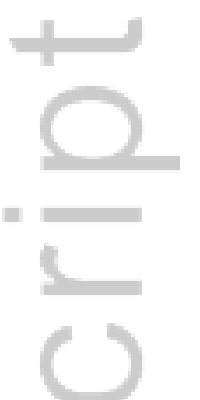

$\infty$
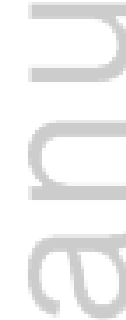

This article is protected by copyright. All rights reserved. 
Figure 9.
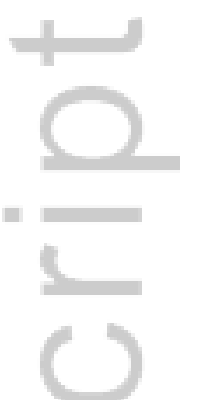

$\infty$
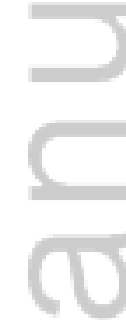

This article is protected by copyright. All rights reserved. 
$0-12$

No SEF
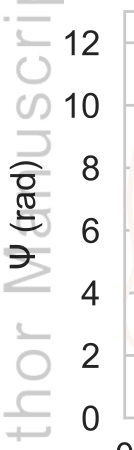
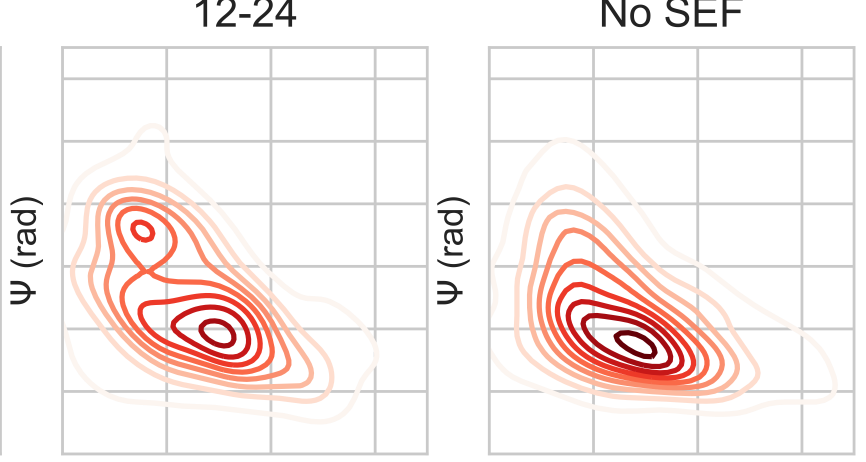

0.015 $-0.013$ - 0.011 - 0.009 - 0.007 - 0.005 - 0.003 $-0.001$ crossing angle $\left({ }^{\circ}\right)$ crossing angle $\left({ }^{\circ}\right)$ crossing angle $\left({ }^{\circ}\right)$ 
VMAX
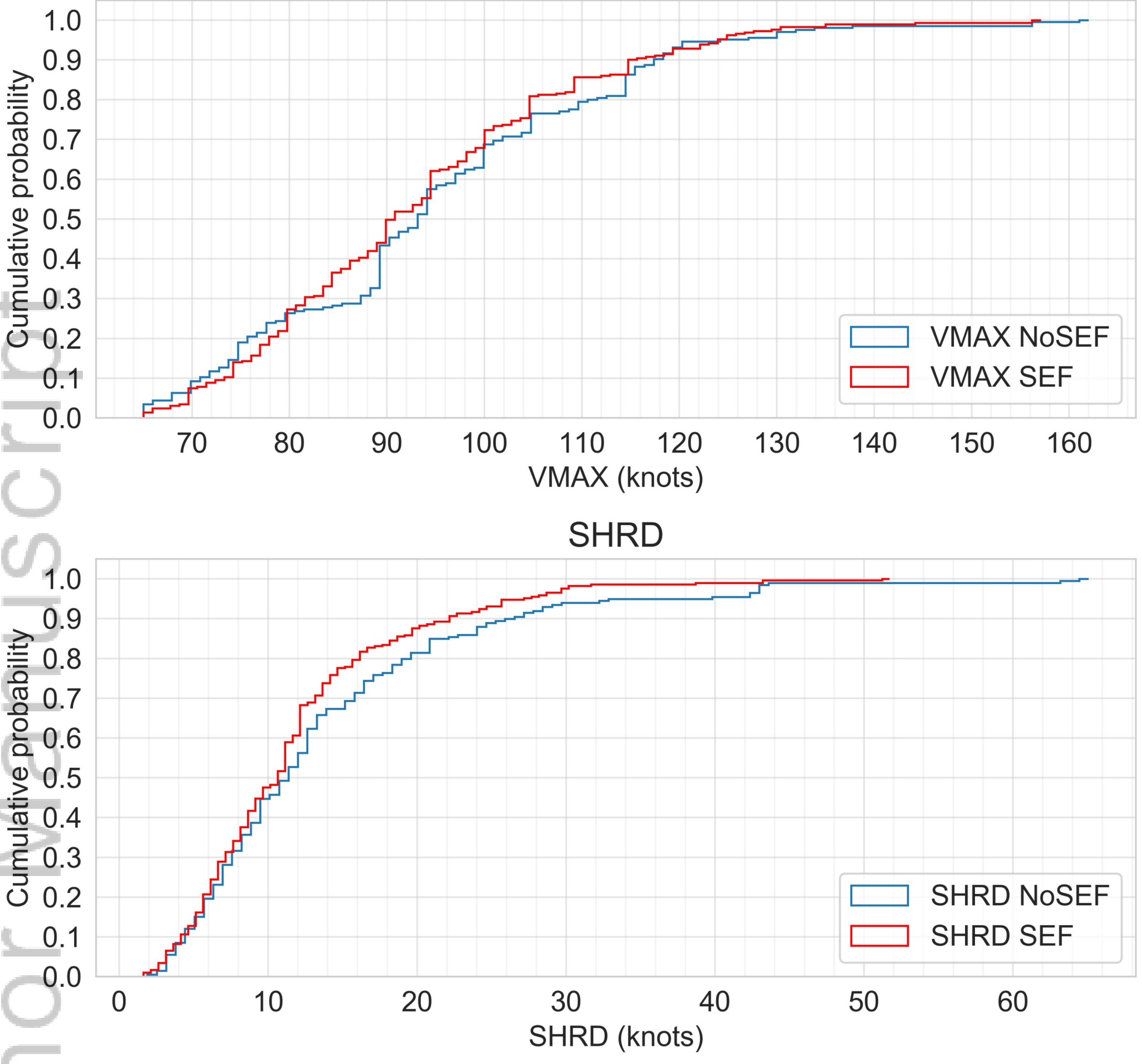

VMPI

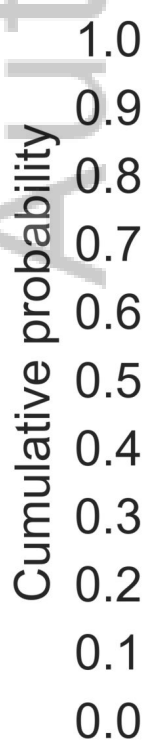


b).

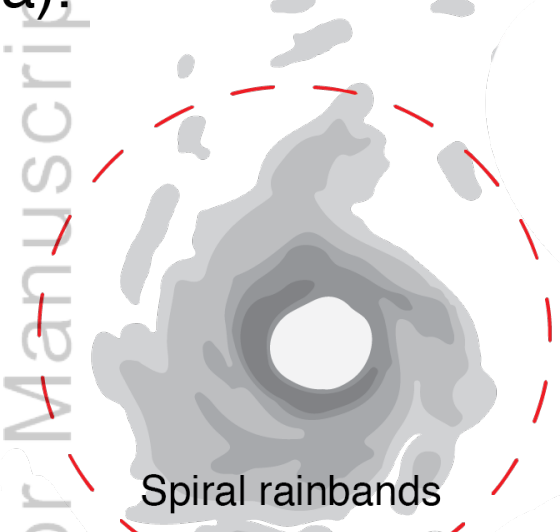

\section{Spiral rainbands}

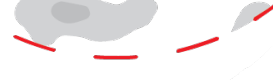

c).

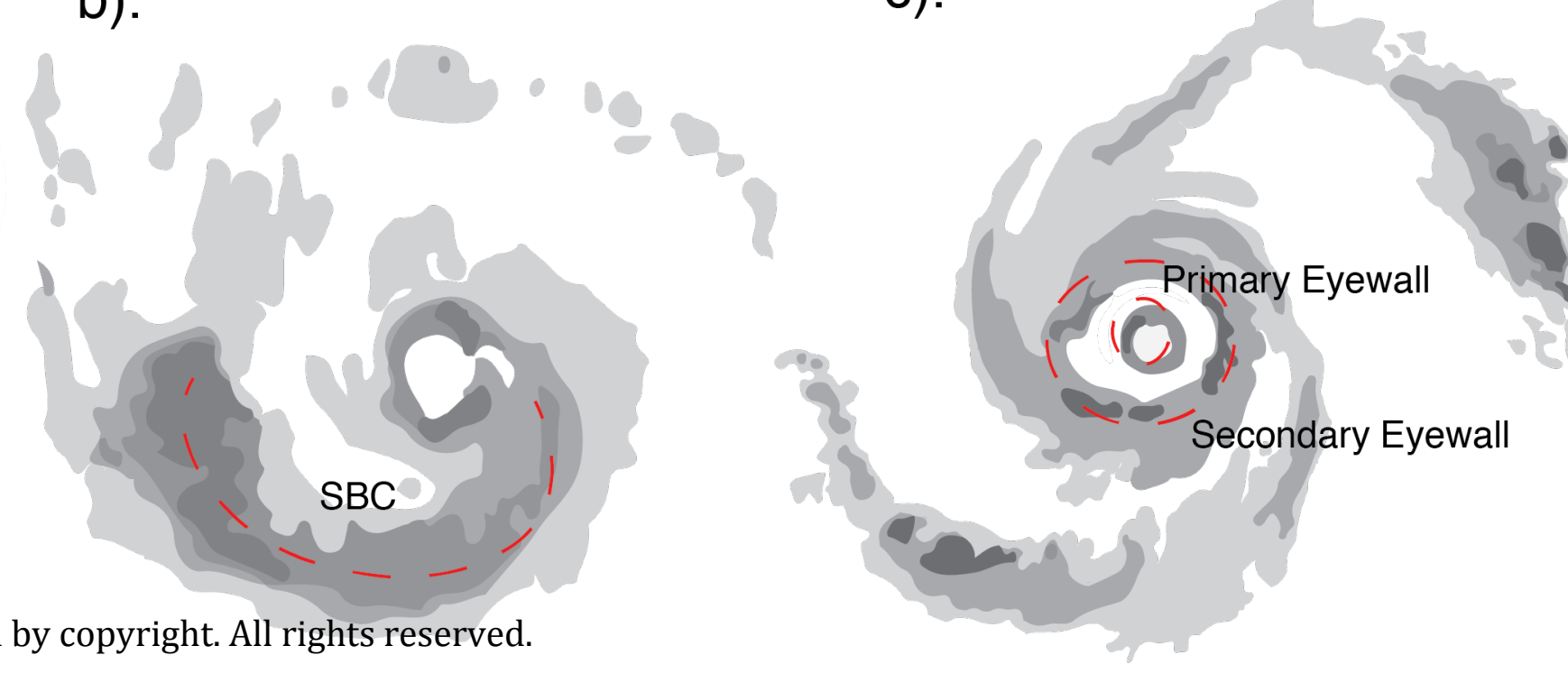




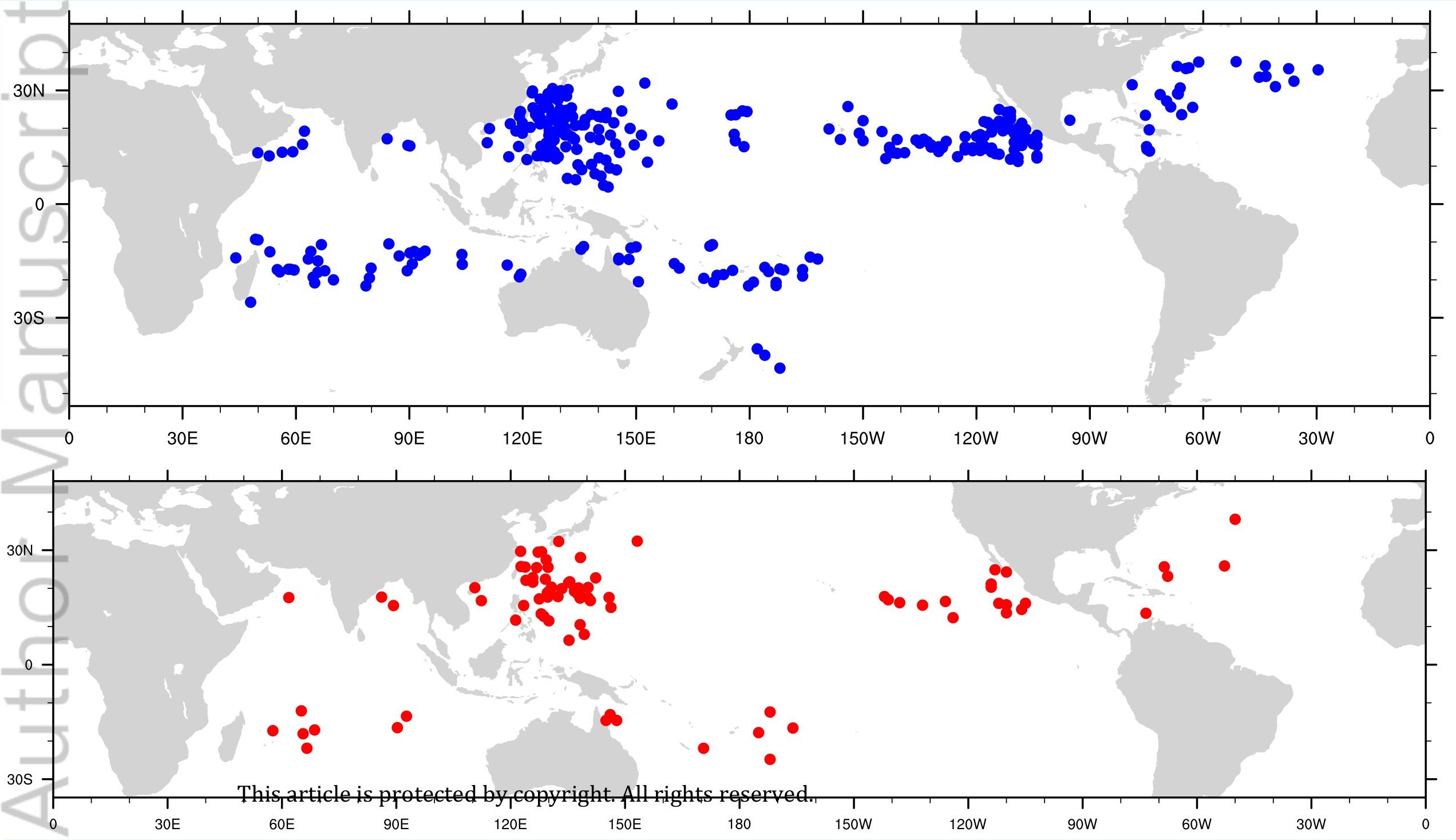


VMAX
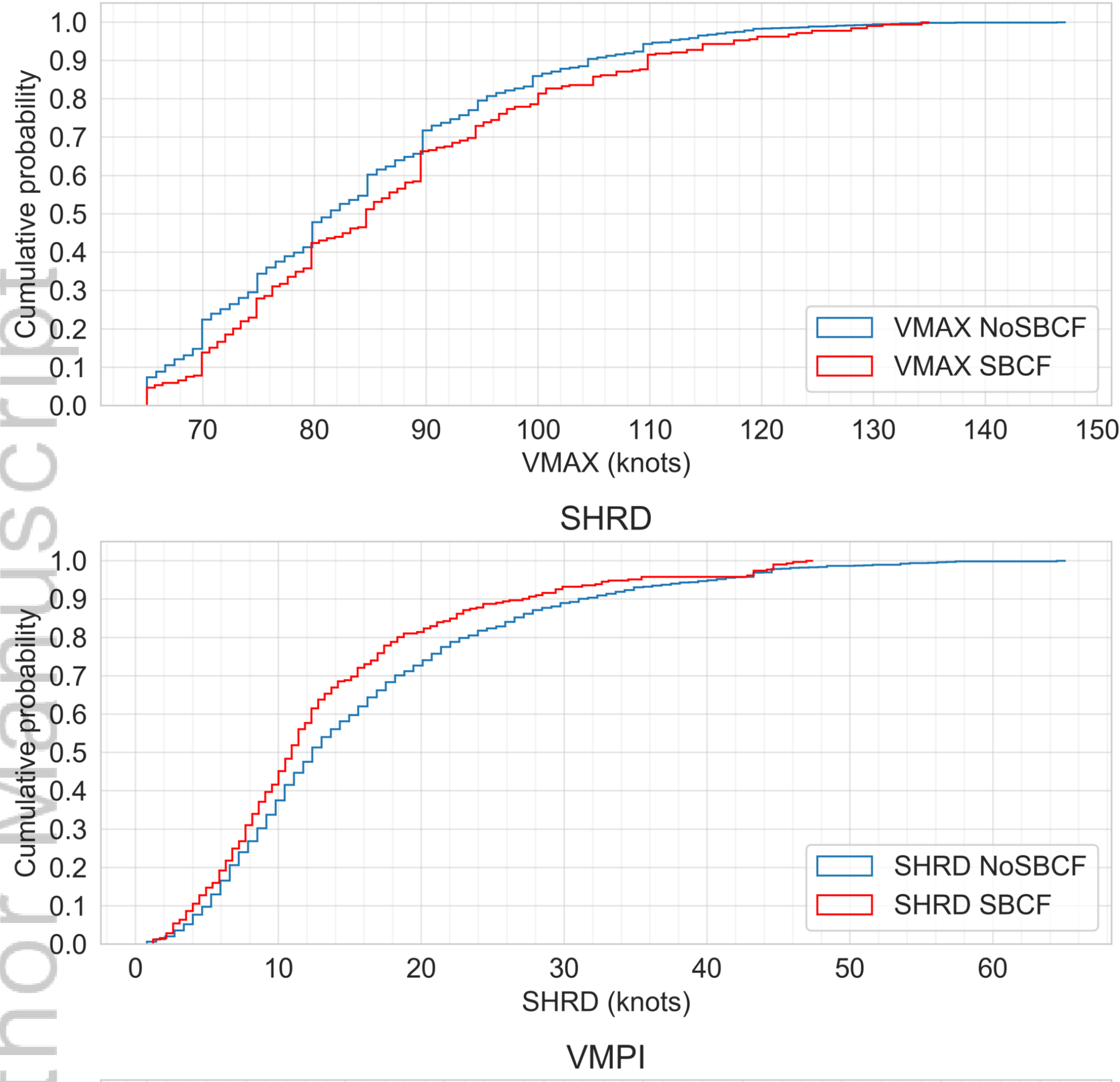

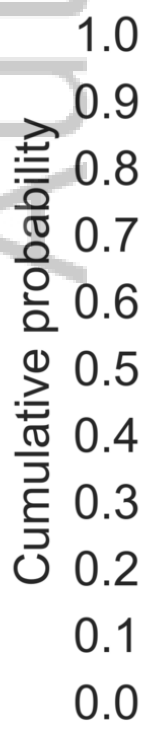
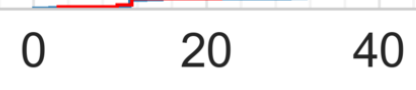

60

This article is protected by copyright. Afrnigtsits reserved. 

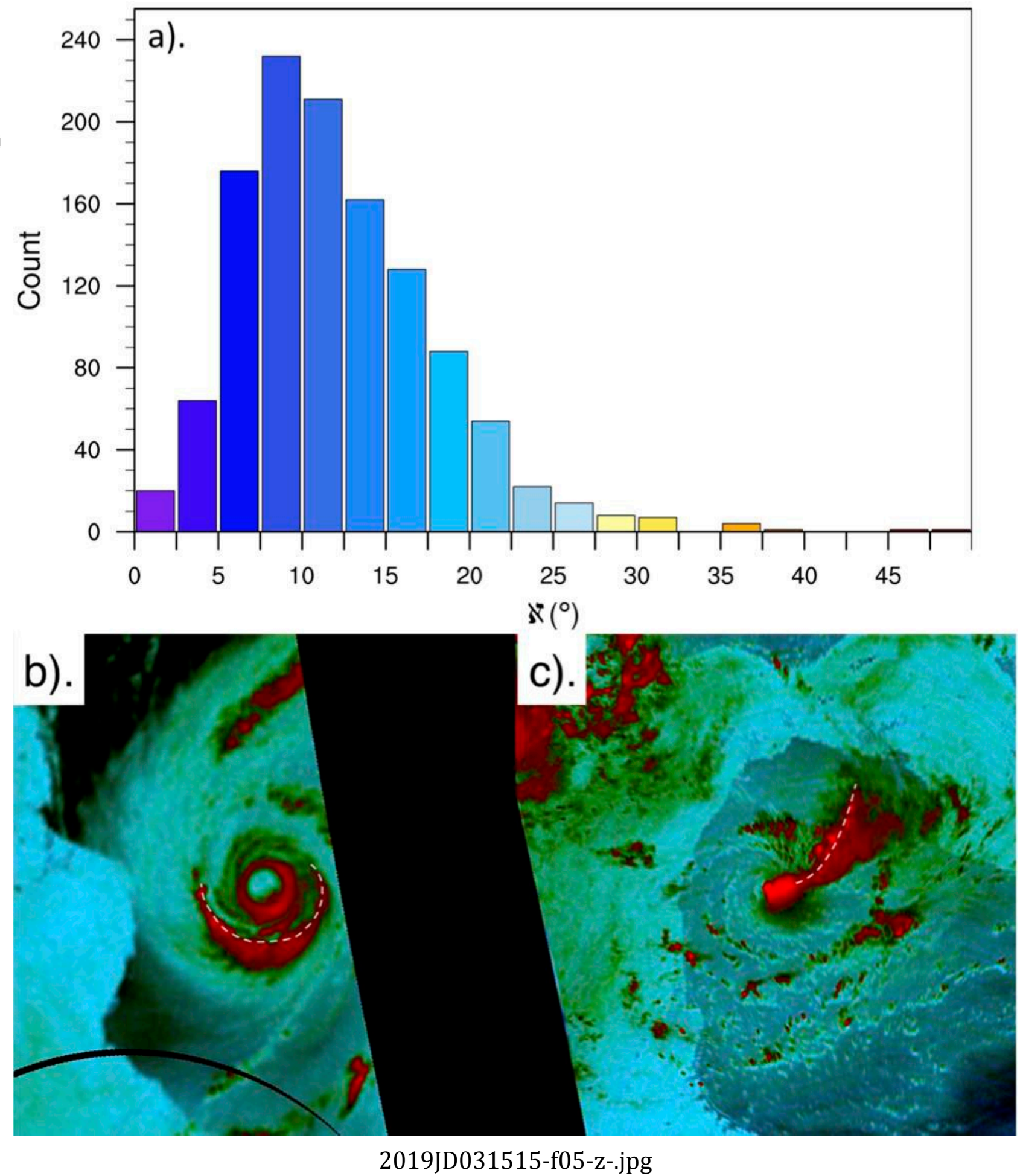

This article is protected by copyright. All rights reserved. 

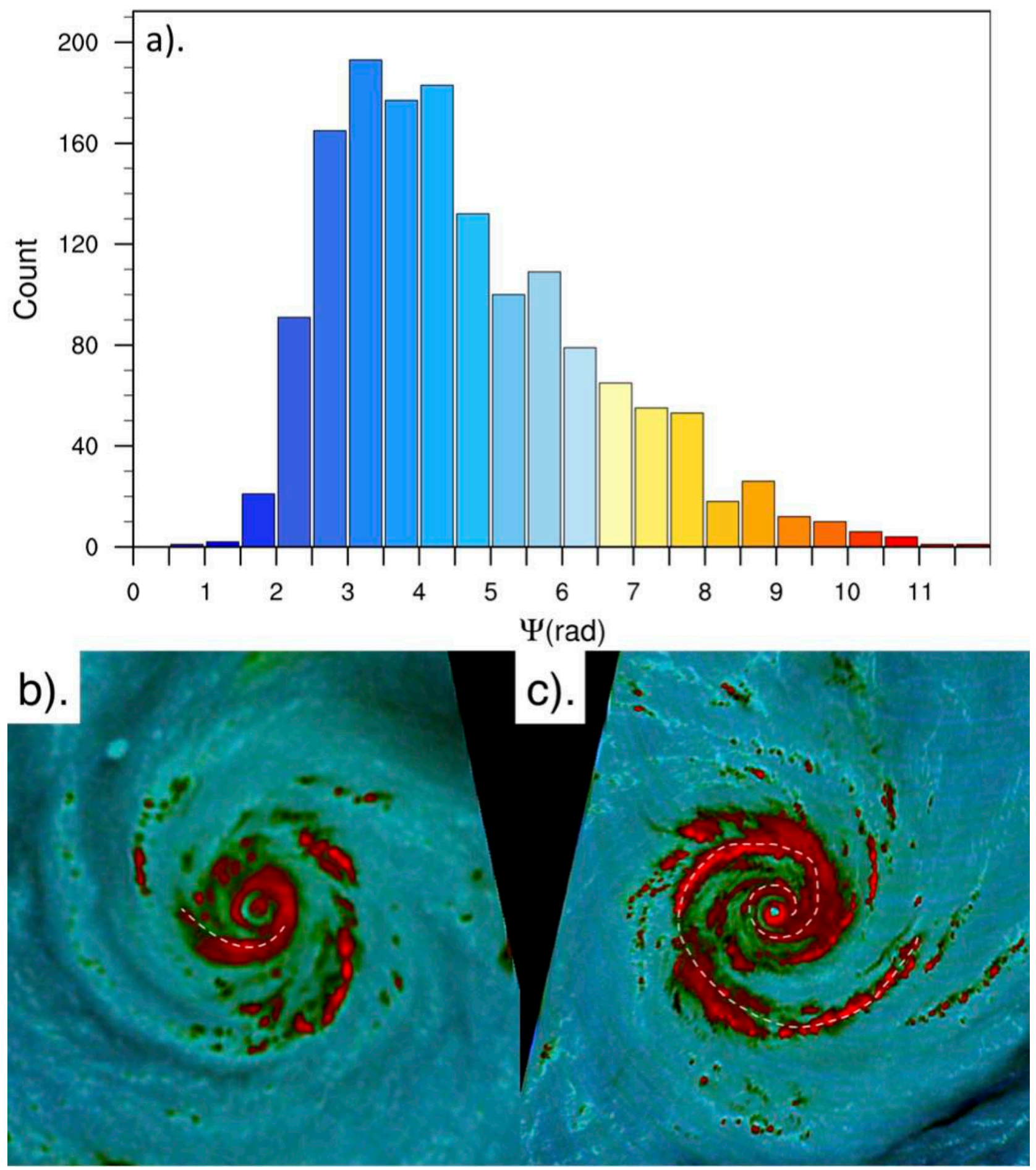

2019JD031515-f07-z-.jpg

This article is protected by copyright. All rights reserved. 
$0-12$

No SEF
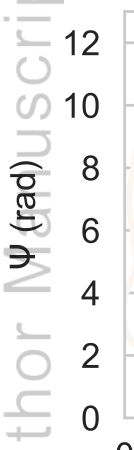
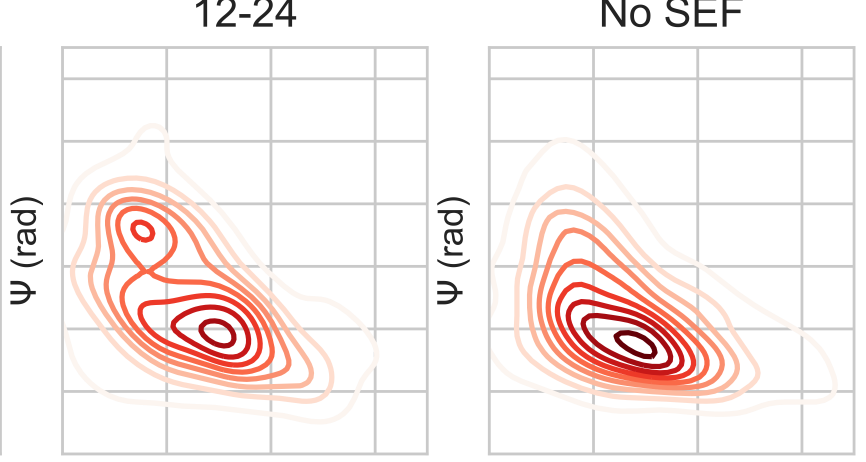

0.015 $-0.013$ - 0.011 - 0.009 - 0.007 - 0.005 - 0.003 $-0.001$ crossing angle $\left({ }^{\circ}\right)$ crossing angle $\left({ }^{\circ}\right)$ crossing angle $\left({ }^{\circ}\right)$ 
VMAX
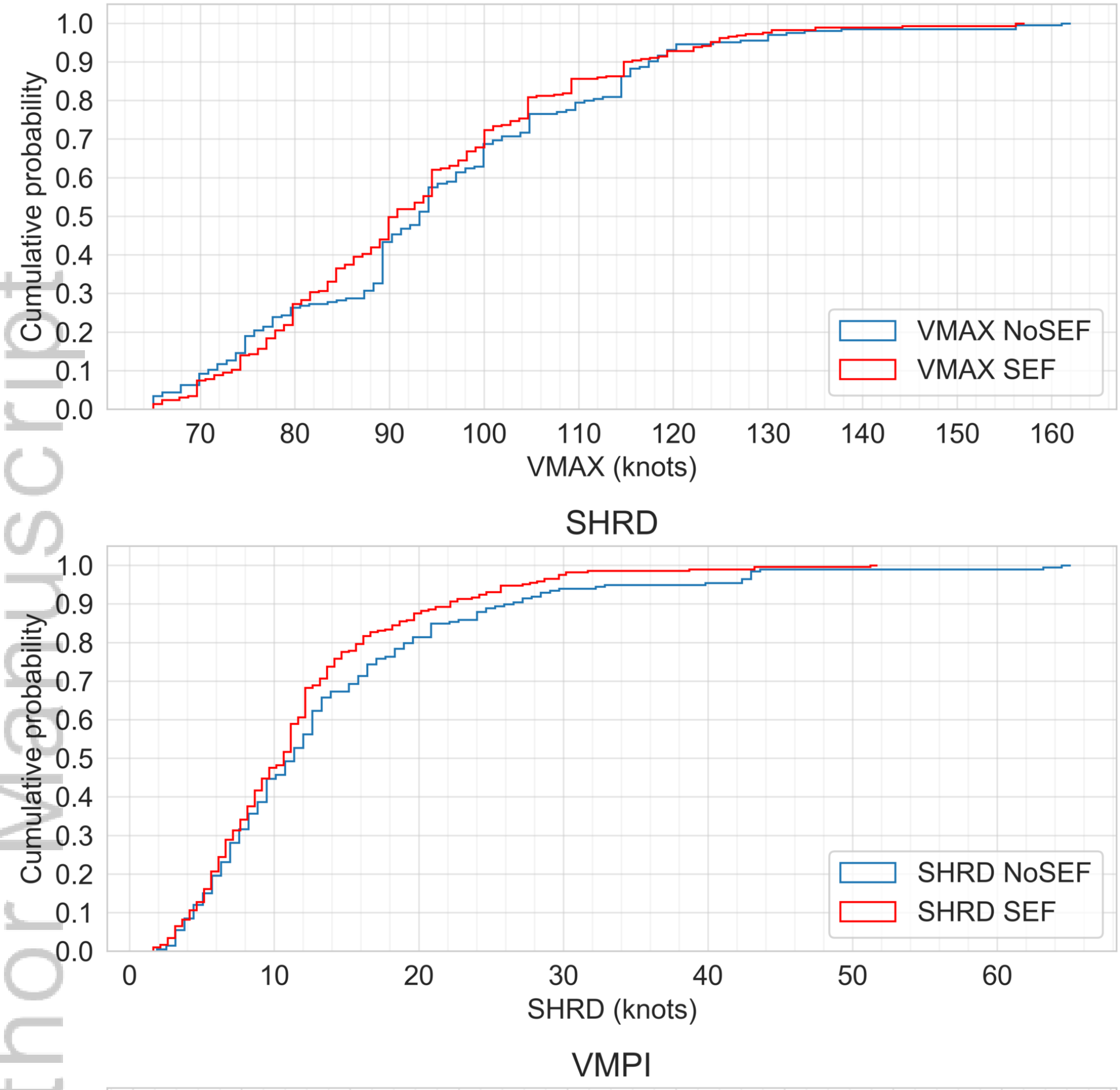

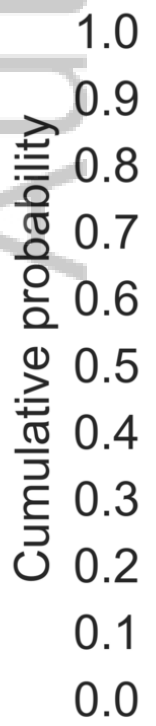

